Original Research Paper

\title{
Asymptotic Properties of Local Sampling on Manifold
}

\author{
Yury Aleksandrovich Yanovich \\ Institute for Information Transmission Problems (Russian Academy of Sciences), Moscow, Russia \\ Faculty of Computer Science, Higher School of Economics (National Research University), Moscow, Russia
}

\author{
Article history \\ Received: 30-06-2016 \\ Revised: 24-08-2016 \\ Accepted: 26-08-2016
}

Email: yyanovich@hse.ru

\begin{abstract}
In many applications, the real high-dimensional data occupy only a very small part in the high dimensional 'observation space' whose intrinsic dimension is small. The most popular model of such data is Manifold model which assumes that the data lie on or near an unknown manifold Data Manifold, (DM) of lower dimensionality embedded in an ambient high-dimensional input space (Manifold assumption about highdimensional data). Manifold Learning is a Dimensionality Reduction problem under the Manifold assumption about the processed data and its goal is to construct a low-di-mensional parameterization of the DM (global low-dimensional coordinates on the DM) from a finite dataset sampled from the DM. Manifold assumption means that local neighborhood of each manifold point is equivalent to an area of low-dimensional Euclidean space. Because of this, most of Manifold Learning algorithms include two parts: 'local part' in which certain characteristics reflecting low-dimensional local structure of neighborhoods of all sample points are constructed and 'global part' in which global low-dimensional coordinates on the DM are constructed by solving certain convex optimization problem for specific cost function depending on the local characteristics. Statistical properties of 'local part' are closely connected with local sampling on the manifold, which is considered in the study.
\end{abstract}

Keywords: Manifold Learning, Asymptotic Expansions, Large Deviations

\section{Introduction}

Many Data Analysis tasks, such as Pattern Recognition, Classification, Clustering, Prognosis, Function reconstruction and others, which are challenging for machine learning problems, deal with real-world data that are presented in high-dimensional spaces and the 'curse of dimensionality' phenomena is often an obstacle to the use of many learning algorithms for solving these tasks.

Fortunately, in many applications, especially in imaging and medical ones, the real high-dimensional data occupy only a very small part in the high dimensional 'observation space' $\mathbb{R}^{p}$ whose intrinsic dimension $\mathrm{q}$ is small (usually, $q \ll p$ ) Donoho (2000; Verleysen, 2003). Thus, various Dimensionality Reduction (Feature extraction) algorithms whose goal is a finding of a low-dimensional parameterization of high-dimensional data can be used as a first key step in solutions of such 'high-dimensional' tasks by transforming the data into their low-dimensional representations (features) preserving certain chosen subject-driven data properties (Bengio et al., 2013;
Bernstein and Kuleshov, 2014; Kuleshov and Bernstein, 2016. Then the low-dimensional features can be used in reduced learning procedures instead of initial high-dimensional vectors avoiding the curse of dimensionality Kuleshov and Bernstein (2014): 'dimensionality reduction may be necessary to discard redundancy and reduce the computational cost of further operations' Lee and Verleysen (2007).

The most popular model of high-dimensional data, which occupy a very small part of observation space $\mathbb{R}^{p}$, is Manifold model in accordance with which the data lie on or near an unknown manifold (Data manifold, DM) $\mathbb{X}$ of lower dimensionality $q<p$ embedded in an ambient high-dimensional input space $\mathbb{R}^{p}$ (Manifold assumption Seung and Lee (2000) about high-dimensional data); typically, this assumption is satisfied for 'real-world' highdimensional data obtained from 'natural' sources. In real examples, a manifold dimension $\mathrm{q}$ is usually unknown and can be estimated by a given dataset randomly sampled from the Data manifold Levina and Bickel (2005; Fan et al., 2009; Einbeck and Kalantana, 2013; Rozza et al., 2011). 
Dimensionality Reduction under the Manifold assumption about the processed data is usually referred to as the Manifold Learning Smith et al. (2009; $\mathrm{Ma}$ and $\mathrm{Fu}, 2011$ ) whose goal is constructing a low-dimensional parameterization of the DM (global low-dimensional coordinates on the DM) from a finite dataset sampled from the DM.

Manifold assumption means that local neighborhood of each manifold point is equivalent to an area of lowdimensional Euclidean space. Because of this, most of Manifold Learning algorithms include two parts: 'local part' in which certain characteristics reflecting lowdimensional local structure of neighborhoods of all sample points are constructed and 'global part' in which global low-dimensional coordinates on the DM are constructed by solving certain convex optimization problem for specific cost function depending on the local characteristics under some normalization constraints (usually, generalized eigenvalues problem). It is typical structure of certain class of manifold learning algorithms such as Locally Linear Embedding (LLE) Roweis and Saul (2000), ISOmerric MAPping (Isomap) Tenenbaum et al. (2000), Laplacian Eigenmaps (LEM) Belkin and Niyogi (2003), Local Tangent Space Alignment (LTSA) Zhang and Zha (2004), Hessian Eigenmaps (HLLE) Donoho and Grimes (2003), Semidefinite Embedding (SDE) Weinberger and Saul (2006) and Diffusion Maps (DFM) Coifman and Lafon (2006).

The radius of the neighborhood should be small enough to achieve small local estimation error. On the other hand, the number of points in the neighborhood should be large enough to get a small statistical error. There are two approaches to choose the ball's size: it consists of the fixed number of neighbors ( $\mathrm{k}$ nearest), or the radius is set. The first case does not guarantee that the radius would be small so that the local approximation error could be large. The distribution of the k-th neighbor is studied in (Levina and Bickel, 2005; Farahmand et al., 2007; Campadelli et al., 2015). Also in Smith et al. (2008) distance to the $\mathrm{k}$-th neighbor assumed to converge to zero and is of the rate of convergence parameter. The second case does not guarantee the large enough number of points if the neighborhood. This question is mentioned in (Levina and Bickel, 2005; Singer and $\mathrm{Wu}, 2012$ ) but it wasn't specifically discussed. Singer and Wu (2012), it was shown that both local and statistical parts of errors are asymptotically small for a specific statistic and also large deviation error was estimated.

Random variable 'the number of points in the neighborhood of a fixed point on manifold' is considered in the present paper. Such random variable is binomial (sum of Bernoulli) and is well studied in general case Shiryayev (1984): De Moivre-Laplace theorem provides its distribution for a fixed success parameter and Poisson theorem provides its distribution for a fixed finite product of success parameters and sample size. In this study, it is supposed that sample is generated from a good enough continuous measure on a good enough unknown manifold and the parameter slowly tends to zero. Thus, the case between de Moivre-Laplace and Poisson theorems is considered: Success parameter tends to zero but it's product with sample size tends to infinity. Another feature of the work is the measure's support: Unknown and curved manifold. In this study, parametric families of random variables (correlated random fields with neighborhood centers as parameters) are studied and uniform results are obtained.

The paper is organized as follows. It is started with the Manifold Learning problem and typical workflow formulation in Section Common Manifold Learning Problem. In Section Results Description, the main results of the paper are listed and commented. In Section Data Model the data model is defined and all assumptions are listed. Then, Main Results Section contains exact formulations of the main results. In Section Some Definitions and Lemmas are listed useful definitions and lemmas to prove main results. Proof of Main Theorems contains the main proofs. In Section Conclusion the paper summary and future work directions are given. In Appendix A. Definitions and lemmas from differential geometry useful definitions and results from differential geometry are recalled. In Section Appendix B. Lemmas Proofs the lemmas from the Section Some Definitions and Lemmas are proved.

\section{Common Manifold Learning Problem}

\section{Manifold Learning as Manifold Embedding}

The main results are strictly formulated Consider unknown -dimensional Data manifold:

$$
\mathbb{X}=\left\{X=f(b) \in \mathbb{R}^{p}: b \in \mathbb{B} \subset \mathbb{R}^{q}\right\}
$$

Covered by a single coordinate chart $\mathbb{B}$ and embedded in an ambient $\mathrm{p}$-dimensional space $\mathbb{R}^{p}, \mathrm{q}<\mathrm{p}$. The chart $\mathbb{B}$ is a one-to-one mapping from open bounded space $\mathbb{B} \subset \mathbb{R}^{p}$ to manifold $\mathbb{X}=f(\mathbb{B})$ with differentiable inverse map $f^{-1}: \mathbb{X} \rightarrow \mathbb{B}$. The manifold intrinsic dimension $\mathrm{q}$ is assumed to be known.

Inverse mapping $h_{f}(X)=f^{-1}(X)$, whose values $b=h_{f}(X) \in \mathbb{B}$ can be considered as low-dimensional coordinates on the manifold $\mathbb{X}$, gives low-dimensional representations (features) $b=h_{f}(X)$ of high-dimensional manifold-valued data $\mathbb{X}$.

If the mappings $h_{f}(X)$ and $f(b)$ are differentiable and $J_{f}(b)$ is $p \times q$ Jacobi an matrix of mapping $f(b)$ than qdimensional linear space:

$T_{X}(\mathbb{X})=\operatorname{Span}\left(J_{f}\left(h_{f}(X)\right)\right)$ 
In $\mathbb{R}^{p}$ is tangent space to the manifold $\mathbb{X}$ at the point $X \in \mathbb{X}$; hereinafter, $\operatorname{Span}(H)$ is linear space spanned by columns of the arbitrary matrix $H$. The tangent spaces can be considered as elements of the Grassmann manifold $\operatorname{Grass}(p, q)$ consisting of all q-dimensional linear subspaces in $\mathbb{R}^{p}$.

Let $\mathbb{X}_{N}=\left\{X_{1}, \ldots, X_{N}\right\} \subset \mathbb{X}$ be a dataset randomly sampled from the DM $\mathbb{X}$ according to certain (unknown) probability measure whose support coincides with $\mathbb{X}$. Common Manifold learning problem is as: Given a sample $\mathbb{X}_{N}$, construct a low-dimensional parameterization of the DM which produces an Embedding mapping:

$$
h: \mathbb{X} \subset \mathbb{R}^{p} \rightarrow \mathbb{Y}_{h}=h(\mathbb{X}) \subset \mathbb{R}^{p}
$$

From the DM $\mathbb{X}$ to the Feature Space (FS) $\mathbb{Y}_{h} \subset \mathbb{R}^{p}, q<p$, which preserves specific properties of the DM.

\section{Manifold Learning as Manifold Embedding}

Following Goldberg et al. (2008), we consider a class consisting of typical Manifold learning algorithms which recover the underlying structure of the Data manifold from the sample; this class includes so-called 'normalized-output' algorithms Goldberg et al. (2008). A common scheme of the considered algorithms is constructed in four steps.

\section{First Step: Neighborhoods Construction}

For each sample point $X_{n}$, local neighborhood $U_{N}\left(X_{n}\right)=\left\{X_{n}, X_{n, 1}, \ldots, X_{n, k(n)}\right\} \subset \mathbb{X}_{N}$ consisting of near sample points is constructed. Typical examples: $U_{N}\left(X_{n}\right)=U_{N}\left(X_{n}, \varepsilon\right)$ consists of sample points that belong to $\varepsilon$-ball in $\mathbb{R}^{p}$ centered at $X_{n}$, or $U_{N}\left(X_{n}\right)=U_{N}\left(X_{n}, k\right)$ in which $k(n)=k$, consists of $k$ nearest-neighbors of the considered point $X_{n}$.

The constructed neighborhoods determine Sample graph $\Gamma\left(X_{N}\right)$ consisting of $N$ vertices $\left\{X_{1}, X_{2}, \ldots, X_{N}\right\}$; the vertices $X_{n}$ and $X_{j}$ are connected by an edge $\left(X_{n}, X_{j}\right)$ when $X_{n} \in U_{N}\left(X_{j}\right)$ and $X_{j} \in U_{N}\left(X_{n}\right)$. These neighborhoods determine 'Euclidean' kernels $K_{\mathrm{E}}\left(X_{n}, X_{j}\right)=I\left(X_{n} \in U_{N}\left(X_{j}\right)\right.$; $\left.X_{j} \in U_{N}\left(X_{n}\right)\right\}$, in which $I(A)$ is an indicator function of the event $A, \quad$ or 'heat-transfer' kernel $K_{h-t}\left(X_{n}, X_{j}\right)=K_{E}\left(X_{n}, X_{j}\right) \cdot \exp \left(-\varepsilon \cdot\left\|X_{n}-X_{j}\right\|\right)$ Belkin and Niyogi (2003).

\section{Second Step: Neighborhoods Descriptions}

Chosen descriptions of the neighborhoods (local descriptions of the DM) are computed. Examples of such descriptions:
- Barycentric coordinates $\left\{w_{n}, 1, w_{n, 2}, \ldots, w_{n, k}\right\}$ of the 'central' point $X_{n}$ with respect to its k nearest-neighbors $\left\{X_{n, 1}, X_{n, 2}, \ldots, X_{n, k}\right\}$ that minimize the reconstruction error functions $\left\|X_{i}-\sum_{j} w_{n, j} X_{n, j}\right\|^{2}$ Roweis and Saul (2000); the neighborhood $U_{N}\left(X_{n}, k\right)$ is used here

- An applying of the Principal Component Analysis (PCA) Jolliffe (2002) to the neighborhood $U_{N}\left(X_{n}, \varepsilon\right)$ results in an $\mathrm{p} \times \mathrm{q}$ orthogonal matrix $Q_{P C A}\left(X_{n}\right)$ whose columns are the PCA principal eigenvectors corresponding to the $\mathrm{q}$ largest PCA eigenvalues (Zhang and Zha, 2004; Bernstein and Kuleshov, 2014). These matrices determine q-dimensional linear spaces $L_{P C A}\left(X_{n}\right)=\operatorname{Span}\left(Q_{P C A}\left(X_{n}\right)\right)$ in the $\mathbb{R}^{p}$ which, under certain conditions, accurately approximate the tangent spaces $T_{\mathbb{X}}\left(X_{n}\right)$ (2) to the $\mathrm{DM} \mathbb{X}$ at the points $X_{n}$ Singer and $\mathrm{Wu}$ (2012).

\section{Third Step: Global Description}

Chosen global description of the DM is computed by solving some convex optimization problems under some normalization constraints. Usually, low-dimensional sample features $\mathbb{Y}_{N}=h\left(\mathbb{X}_{N}\right)=\left\{y_{1}, y_{2}, \ldots, y_{N}\right\} \subset \mathbb{Y}_{h} \quad$ are computed by minimization of chosen cost function $L\left(\mathbb{Y}_{N} \mid \mathbb{X}_{N}\right)$ over $\mathbb{Y}_{N}$. Examples of cost functions:

$$
\begin{aligned}
& L_{L L E}\left(\mathbb{Y}_{N} \mid \mathbb{X}_{N}\right)=\sum_{n=1}^{N}\left\|y_{n}-\sum_{j} w_{n, j} y_{n, j}\right\|_{F}^{2} \\
& L_{L E}\left(\mathbb{Y}_{N} \mid \mathbb{X}_{N}\right)=\sum_{n=1}^{N}\left\|y_{n}-y_{j}\right\|_{F}^{2} \\
& L_{L T S A}\left(\mathbb{Y}_{N} \mid \mathbb{X}_{N}\right)=\sum_{n=1}^{N} \|\left(I_{q}-Q_{P C A}\left(X_{n}\right) \cdot Q_{P C A}\left(X_{n}\right)^{T}\right) \\
& \cdot H_{n} \cdot \mathbb{Y}_{(n)} \|_{F}^{2}
\end{aligned}
$$

Are used in the algorithms LLE Roweis and Saul (2000), LE Belkin and Niyogi (2003) and LTSA Zhang and Zha (2004), respectively; here $q \times(k(n)+1)$ matrix $Y_{n}$ consists of q-dimensional columns $\left\{y_{n}, y_{n, 1}\right.$, $\left.y_{n, 2}, \ldots, y_{n, k(n)}\right\}$ in which the same pairs of indices $n, j$ are uses as in $U_{N}\left(X_{n}\right), H_{n}=I_{q^{-}}(1 / k(n)) \times 1 \times 1^{T}$ is $\mathrm{q} \times \mathrm{q}$ centering matrix in which $I_{q}$ is $\mathrm{q} \times \mathrm{q}$ unit matrix and qdimensional vector 1 consists of units. Some normalization constraints on the Feature sample $\mathbb{Y}_{N}$ are used to avoid the degenerate solutions.

ISOMAP Tenenbaum et al. (2000) is based on estimating the geodesic curves on the DM $\mathbb{X}$. Consider the sample weighted graph $\Gamma_{W}\left(\mathbb{X}_{N}\right)$, in which an edge $\left(X_{n}, X_{j}\right)$ has weight $\left\|X_{n}-X_{j}\right\|$. Let $\left\{D_{n j}\right\}$ are the lengths of the shortest paths between the vertices $X_{n}$ and $X_{j}$ in the graph $\Gamma_{W}\left(\mathbb{X}_{N}\right)$ that can be computed with using the Dijkstra algorithm; these quantities $\left\{D_{n j}\right\}$ accurately estimate geodesic distances between the points $X_{n}$ and $X_{j}$ 
on the DM $\mathbb{X}$ Tenenbaum et al. (2000). After that, the Feature sample $\mathbb{Y}_{N}$ are computed with use MultiDimensional Scaling framework (MDS) Cox and Cox (2008) by minimization over $\mathbb{Y}_{N}$ the MDS cost function

$$
L_{\text {ISOMAP }}\left(\mathbb{Y}_{N} \mid \mathbb{X}_{N}\right)=\sum_{n, j=1}^{N}\left\|D_{n j}-\right\| y_{n}-y_{j}\|\|^{2} \text {. }
$$

\section{Fourth Step: Out-of-Sample Extension}

The Feature sample $\mathbb{Y}_{N}$ gives the values of the Embedding mapping $h(X)$ (3) only at sample points; a finding of low-dimensional features $h(X)$ for Out-ofSample (OoS) points $X \in \mathbb{X} \backslash \mathbb{X}_{N}$ is usually called OoSextension problem. The OoS-extension for the algorithms LLE Roweis and Saul (2000), ISOMAP Tenenbaum et al. (2000), LE Belkin and Niyogi (2003), has been found in Bengio et al. (2003) with use Nyström's eigendecomposition technique Saul et al. (2003).

\section{Results Description}

The main results are strictly formulated in Section Main Results and all the assumptions, used in the proof, strictly formulated in Section Data Model. Some substantive comments are given here.

\section{Data Model}

Data Model consists of assumptions about support (manifold), assumptions about sample distribution and assumptions about neighborhood parameter. The paper deals with 'good enough' manifolds with known dimensionality q. The problem of dimensionality estimation is a problem of the only integer parameter estimation and solutions Campadelli et al. (2015) with the rate of error probability $\sim \exp (-C . N)$ are known, where $\mathrm{N}$ is a sample size $\mathrm{C}>0$ is constant. Such rate is smaller than the rates in this article. The sample assumed to be independent identically distributed (i.i.d.) with unknown 'good' continuous measure on the manifold. The neighborhood parameter slowly tends to zero.

\section{Main Results}

Manifold behaves as a linear subspace in a small neighborhood of a point. Therefore, the intersection of a full dimensional Euclidean ball with a manifold is close to the low dimensional ball. Thus, the number of sampling points, fallen into the neighborhood, should be proportional to the volume of the q-dimensional ball. This result was mentioned a number of times in the works (for example, Levina and Bickel (2005; Einbeck and Kalantana, 2013; Singer and Wu, 2012)). However, in the Theorem 1, we prove that the number of sampling points in the neighborhood, divided by the volume of the neighborhood, is a consistent estimate of the density at the point. The Theorem 2 prove that conditional distribution of sample points in the neighborhood is asymptotically uniform. The Corollary 1 sets that all directions from tangent space are equal for the conditional distribution. So, one could think of conditional distribution as of uniform distribution on the ball in tangent space. In the Theorem 3 asymptotic expansion of the considered statistics is given and in the Theorem 4 assesses the probability of large deviations. The Theorem 5 prove a uniform result of the large deviations probability: If we consider all points of the manifold, which are a little removed from the border, as the centers of the balls, then the minimum over all balls of points in each of them, will be asymptotically infinitely large with a high probability.

The features of the results are the curvature of the unknown sample support, the tendency to zero of the random variable mathematical expectation ('hit one sampling point in a ball with decreases to zero radius'), the need to obtain uniform estimation on the manifold.

The basic ideas used in proofs: Local linearization of the support, a generalization of de Moivre-Laplace theorems to the case of decreasing the probability of success in the Bernoulli scheme, the use of inequalities for the probabilities of large deviations of sums of i.i.d. random variables, the use of finite nets.

\section{Data Model}

Let's assume that:

M1. $\mathbb{X} \subset \mathbb{R}^{p}$ is a q-dimensional manifold covered with a single map. That is, for some open $\mathbb{B} \subset \mathbb{R}^{q}$ and $f: \mathbb{B} \rightarrow \mathbb{R}^{p}: \mathbb{X}=f(\mathbb{B})$ homeomorphism

M2. $\mathrm{q}$ is known

M3. $\mathbb{B}$ is a bounded set

M4. Eigenvalues of $\mathrm{q} \times \mathrm{q}$ Jacobi matrixes product $J_{f}^{T}(b) \cdot J_{f}(b)$ of $f$ mapping uniformly separated from 0 and infinity

M5. Hessian of $f$ mapping exists and bounded on $\mathbb{X}$

M6. Third order derivatives of $f$ existing and are elementwise bounded on $\mathbb{X}$

M7. Condition number $c(\mathbb{X})$ is bounded, where $\mathbb{X}$ is the smallest number such that for each point, which is distant from $\mathbb{X}$ at a distance smaller than $1 / c(\mathbb{X})$, the only projection exists on $\mathbb{X}$ Niyogi et al. (2008)

M8. Manifold $\mathbb{X}$ is geodesically convex, that is, for any pair of points on $\mathbb{X}$ exists geodesic line and it is the shortest path

Note 1. The assumption M1 is equivalent to
the existence of the global dimension
coordinate system on the manifold.
Assumption $M 3$ is used to obtain uniform
properties of statistics.

Note 1. The assumption M1 is equivalent to the existence of the global dimension Assumption M3 is used to obtain uniform properties of statistics. 


\begin{abstract}
Assumptions M4-M6 represent the conditions of smoothness and are used to connect Euclidean distances and volumes with the distances and the volumes on the manifold. Property $M 7$ means that the manifold does not contain a 'short-circuit': The closeness of the points on the Euclidean distance implies the closeness of points on the manifold. That is, the intersection of the small Euclidean neighborhood of the point creates a small neighborhood of a point on the manifold.

The assumption M8 is a technical simplification for Taylor expansion.
\end{abstract}

The set $\mathbb{B}$ is bounded and derivatives of $f$ are bounded too, so the manifold $\mathbb{X}$ is also bounded. Let a be an edge of the circumscribed cube of manifold:

$$
a=\inf \left\{a^{\prime}: a^{\prime}, a_{1}, \ldots, a_{p}: \mathbb{X} \subset \bigotimes_{i=1}^{p}\left[a_{i}, a_{i}+a^{\prime}\right]\right\}
$$

Let $C_{J}$ and $C_{j}$ be the minimum and the maximum eigenvalues of the metric tensor $J_{f}(b)^{T} J_{f}(b), b \in \mathbb{B}$ correspondingly:

$$
\begin{aligned}
& c_{J}=\inf _{b \in \mathbb{B}} \min _{\lambda \in \sigma\left(J_{f}(b)^{T} J_{f}(b)\right)} \lambda \\
& C_{J}=\inf _{b \in \mathbb{B}} \max _{\lambda \in \sigma\left(J_{f}(b)^{T} J_{f}(b)\right)} \lambda
\end{aligned}
$$

Let $C_{H}$ be the maximum element of Hessian matrix of $(\mathbb{B}, f)$ mapping:

$C_{H}=\sup _{X \in \mathbb{X}, i, j=1, \ldots, q}\left\|\frac{\partial^{2} f(b)}{\partial b_{i} \partial b_{j}}\right\|=\sup _{X \in \mathbb{X}, i, j=1, \ldots, q} \sqrt{\sum_{k=1}^{p}\left|\frac{\partial^{2} f_{k}(b)}{\partial b_{i} \partial b_{j}}\right|^{2}}$

where, $X=\left(f_{1}(b), \ldots, f_{p}(b)\right)^{\mathrm{T}}$. Let:

$$
\begin{aligned}
& C_{T}=\sup _{X \in \mathbb{X}, i, j, m=1, \ldots, q} \| \frac{\partial^{3} f(b)}{\partial b_{i} \partial b_{j} \partial b_{m}} \mid \\
& =\sup _{X \in \mathbb{X}, i, j, m=1, \ldots, q} \sqrt{\sum_{k=1}^{p}\left|\frac{\partial^{3} f_{k}(b)}{\partial b_{i} \partial b_{j} \partial b_{m}}\right|^{2}}
\end{aligned}
$$

Manifold $\mathbb{X}$ is unknown and it is represented by finite random sample $\mathbb{X}_{n}=\left\{X_{1}, \ldots, X_{N}\right\} \subset \mathbb{X} \subset \mathbb{R}^{p}$ with $N$ points. Moreover, it is assumed about sample selection that:

S1. Points from $\mathbb{X}_{n}$ are i.i.d. with some probability measure $\mu$ such that $\mathbb{X}$ is its support: $\mathbb{X}=s u p p \mu$

S2. Measure $\mu$ is continuous with respect to Riemannian measure on manifold and its density $p_{\mu}$ is bounded from zero and infinity
S3. Density $p_{\mu}$ is twice smooth on $\mathbb{X}$ and its derivatives are bounded

Note 2. Manifold $\mathbb{X}$ has Riemannian measure $d V(X)$ (volume measure) which is equal to the -dimensional volume in the main term (Section Some Definitions and Lemmas). Let $(\Omega, \mathfrak{B}, \mathbb{P})$ be a probability space, than Borelian function, $\mathfrak{B} \rightarrow \mathbb{X}: X=X(\omega)$ is called random variable on the manifold. Let's call such induced on $\mathbb{X}$ the measure as $\mu$. If for $\begin{array}{llr}\text { each } \quad \text { Borelian } & \text { set } \quad \begin{array}{r}\mathfrak{X} \in \mathfrak{B} \\ \mu(X \in \mathfrak{X})=\int_{\mathfrak{X}} p_{\mu}(X) d V(X),\end{array} \text { then the }\end{array}$ function $p_{\mu}(X), X \in \mathbb{X}$ is a probability density function Pennec (1999).

Let $p_{\min }$ and $p_{\max }$ be the minimum and maximum values of $p_{\mu}$ (they exist by $\mathrm{S} 2$ ):

$$
\begin{aligned}
& p_{\min }=\inf _{X \in \mathbb{X}} p_{\mu}(X) \\
& p_{\max }=\sup _{X \in \mathbb{X}} p_{\mu}(X)
\end{aligned}
$$

Let define the bounds for maximum eigenvalues of first and second derivatives (exist by S3):

$$
\begin{aligned}
& C_{p, 1}=\sup _{X \in \mathbb{X}, \theta \in T_{X}(\mathbb{X}):\|\theta\|=1}\left\|\nabla_{\theta} p_{\mu}(X)\right\| \\
& C_{p, 2}=\sup _{X \in \mathbb{X}, \theta \in T_{X}(\mathbb{X}):\|\theta\|=1}\left\|\nabla_{\theta} \nabla_{\theta} p_{\mu}(X)\right\|
\end{aligned}
$$

where, $\nabla_{\theta}$ is a covariant derivative (Appendix A), which is a kind of directional derivative generalization for the manifold.

Note 3. S1 and S2 are standard assumptions whose guarantee correspondence between sample $\mathbb{X}_{N}$ and manifold $\mathbb{X}$. Assumption S3 is useful for uniform results.

For neighborhood parameter $\varepsilon=\varepsilon(\mathrm{N})$ it is assumed that:

- P1. For $N \rightarrow \infty: \varepsilon \rightarrow 0$

- P2. For $N \rightarrow \infty: N \varepsilon^{q} \rightarrow \infty$

- P3. For $N \rightarrow \infty: N \varepsilon^{q+4} \rightarrow 0$

Note 4. The assumption P1 means that the neighborhood size tends to zero and therefore the expansion of functions at the main term is a term with the lowest degree of length. The assumption $P 2$ provides an infinite number of sample points in the neighborhood despite the 
decrease in the size of the neighborhood. Assumption P3 is stronger than P1 and guarantees that the contribution of the bias of the order $\varepsilon^{2}$ is infinitely small in the results of the central limit theorem for the number of points of order $N \varepsilon^{q}$.

\section{Main Results}

Let $I_{\varepsilon}\left(X_{n} \mid X\right)$ be an indicator of the event ' $\mathrm{p}$ dimensional distance between $X_{n}$ and $X$ is less of equal to $\varepsilon$, that is $I_{\varepsilon}\left(X_{n} \mid X\right)$ for $\left\|X_{n}-X\right\|<\varepsilon$ and $I_{\varepsilon}\left(X_{n} \mid X\right)=0$ otherwise.

Let $N_{\varepsilon}(X)$ be the number of sample $\mathbb{X}_{N}$ points in pdimensional $\varepsilon$-neighborhood of $X \in \mathbb{R}^{p}$ :

$N_{\varepsilon}(X)=\sum_{n=1}^{N} I_{\varepsilon}\left(X_{n} \mid X\right)$

Let $V_{q}$ be the volume of q-dimensional ball of a unit radius:

$V_{q}=\frac{\pi^{q / 2}}{\Gamma(q / 2+1)}$

where, $\Gamma(z)=\int_{0}^{\infty} t^{z-1} e^{-t} d t, z>0$ is a gamma function, that is $V_{2 m}=\frac{\pi^{m}}{m !}$ and $V_{2 m+1}=\frac{2^{m} \pi^{m}}{(2 m+1) ! !}$ for integer $\mathrm{m}$.

Theorem 1. (weak law of large numbers). If M1-M8, S1-S3 and P1-P2, then for each $X \in \mathbb{X}$ and $N_{\varepsilon}(X)$ for $N \rightarrow \propto$ :

$$
\frac{N_{\varepsilon}(X)}{N \varepsilon^{q}} \stackrel{p}{\longrightarrow} p_{\mu}(X) \cdot V_{q}
$$

where, $V_{q}$ is a volume of -dimensional ball (14).

Theorem 2. (conditional uniform distribution). If M1-M8, S1-S3 and P1-P2, then for each $X \in \mathbb{X}$ for $N \rightarrow \propto$ :

$$
p\left(X_{n} \mid I_{\varepsilon}\left(X_{n} \mid X\right)=1\right) \cdot \varepsilon^{q} \rightarrow 1 / V_{q}
$$

where, $V_{q}$ is a volume of q-dimensional ball (14).

Corollary 1. If M1-M8, S1-S2 and P1-P2, then for each $X \in \mathbb{X}$ the spectrum of a local covariance matrix

$$
\Sigma=\frac{\sum_{n=1}^{N} I_{\varepsilon}\left(X_{n} \mid X\right) \cdot\left(X_{n}-X\right) \cdot\left(X_{n}-X\right)^{T}}{\sum_{n=1}^{N} I_{\varepsilon}\left(X_{n} \mid X\right)} \in \mathbb{R}^{p \times p} \text { tends to ones }
$$

and $\mathrm{p}$-q zeros as $N \rightarrow \propto$. Moreover, ones correspond to vectors from tangent space and zeros corresponds to vectors from cotangent space.
Theorem 3. (central limit theorem for $\left.N_{\varepsilon}(X)\right)$. If M1M8, S1-S3 and P1-P3, then for each $X \in \mathbb{X}$ and $N_{\varepsilon}(X)$ (13) $N \rightarrow \propto$ :

$$
\frac{N_{\varepsilon}(X)-N \varepsilon^{q} p_{\mu}(X) V_{q}}{\sqrt{N \varepsilon^{q} p_{\mu}(X) V_{q}}} \stackrel{d}{\longrightarrow} N(0,1)
$$

where, $V_{q}$ is a volume of q-dimensional ball $(14), N(0,1)$ is standard normal distribution.

Theorem 4. (large deviations of $\left.N_{\varepsilon}(X)\right)$. If M1-M8, $\mathrm{S} 1-\mathrm{S} 2$ and P1-P3, then for $0<z<1 / 16$ :

$$
\begin{aligned}
& P\left(\frac{N_{\varepsilon}(X)}{N \varepsilon^{q} V_{q}} \leq p_{\text {min }} \cdot(1-z)-\varepsilon^{2} \cdot \frac{C_{\mathbb{E}} p_{\max }}{V_{q} p_{\text {min }}}\right) \\
& \leq \exp \left(-4 z^{2} \cdot N \varepsilon^{q} V_{q} p_{\text {min }}^{2} / p_{\text {max }}\right)
\end{aligned}
$$

where, $V_{q}$ is a volume (14), $C_{\mathbb{E}}$ is a constant from Corollary 4 , constants $p_{\min }, p_{\max }, C_{R i c}, C_{p, 1}$ and $C_{p, 2}$ are defined in (9), (10), Lemma 4, (11), (12) correspondingly.

If also $\mathrm{S} 3$, then for $0<\mathrm{z}<1 / 16$ :

$$
\begin{aligned}
& P\left(\frac{N_{\varepsilon}(X)}{N \varepsilon^{q} V_{q} p_{\mu}(X)}-1 \geq z+\varepsilon^{2} \cdot \frac{C_{\mathbb{E}}}{V_{q} p_{\mu}(X)}\right) \\
& \leq \exp \left(-4 z^{2} \cdot N \varepsilon^{q} V_{q} p_{\mu}(X)\right) ; \\
& \leq P\left(\frac{N_{\varepsilon}(X)}{N \varepsilon^{q} V_{q} p_{\mu}(X)}-1 \leq-z-\varepsilon^{2} \cdot \frac{C_{\mathbb{E}}}{V_{q} p_{\mu}(X)}\right) \\
& \leq \exp \left(-4 z^{2} \cdot N \varepsilon^{q} V_{q} p_{\mu}(X)\right) .
\end{aligned}
$$

Theorem 5. (uniform large deviation). If M1-M8, S1S2 and P1-P3, then for $\varepsilon \leq \min \left\{\frac{1}{c(\mathbb{X})}, \sqrt{\frac{24(\sqrt[q]{2}-1)}{\max \left\{1, C_{I I}\right\}}}, \frac{1}{2 \sqrt{C_{R i c}}}, \frac{1}{\sqrt{C_{R i c} C_{p, 2}}}\right.$, $\left.\frac{1}{\sqrt{C_{R i c} C_{p, 1}+C_{p, 2}+1}}, \frac{1}{2}, \frac{1}{\sqrt{2 C_{\mathbb{E}}}}, \sqrt[q]{\frac{1}{4 V_{q} p_{\min }}}, \sqrt[q]{\frac{1}{4 p_{\text {min }}}}\right\}$, each point (not too close to the boundary) $X \in \mathbb{X}_{\varepsilon}$ (17) and:

$$
\begin{aligned}
& P\left(\inf _{X \in \mathbb{X}_{\varepsilon}} \frac{N_{\varepsilon}(X)}{N \varepsilon^{q} V_{q}} \leq p_{\min } \cdot(1-z)-\varepsilon^{2} \cdot \frac{C_{\mathbb{E}} p_{\max }}{V_{q} p_{\min }}\right) \\
& \leq\left(\frac{6 a \sqrt{p}}{\varepsilon}\right)^{p} \exp \left(-4 z^{2} \cdot N \varepsilon^{q} V_{q} p_{\text {min }}{ }^{2} /\left(9 p_{\max }\right)\right)
\end{aligned}
$$

where, $V_{q}$ is a volume (14), $C_{\mathbb{E}}$ is a constant from Corollary 4 , constants $p_{\min }, p_{\max }, C_{R i c}, C_{p, 1}$ and $C_{p, 2}$ and are defined in (9), (10), Lemma 4, (11), (12) correspondingly and $\mathrm{a}>0$ is an edge of the circumscribed other cube (4). 
Note 5. If $\varepsilon(N) \geq C_{\alpha} \cdot N^{-\alpha}, \alpha<1 / q, C_{\alpha}>0$, then the right part of Theorem's 4 equation tends to zero faster than any degree $N^{-\beta}$, $\beta>0$ as a product of exponential and polynomial terms.

\section{Some Definitions and Lemmas}

Definitions and Lemmas which are used to prove main theorems are listed in this section.

\section{Local Linearization}

Manifold $\mathbb{X}$ is close to q-dimensional linear tangent space $T_{X}(\mathbb{X})$ in a neighborhood of each point. Differentiation on the manifold is slightly different from the differentiation in Cartesian coordinates in $\mathbb{R}^{k}$. The differences are mainly of a technical nature and the main difference lies in the fact that derivatives are defined only in the directions from $T_{X}(\mathbb{X})$ (Appendix A).

In a sufficiently small neighborhood of $X \in \mathbb{X}$ there exists a one-to-one mapping between the points of the manifold $\mathbb{X}$ and a subset of the elements of the tangent space $T_{X}(\mathbb{X})$. In this neighborhood vectors $V \in T_{X}(\mathbb{X})$ are coordinates. These coordinates are called locally Riemann (Appendix A).

Manifold volume element and q-dimensional volume element of the tangent space are related by the following lemma:

Lemma 1. (Petersen (2006)) Riemannian measure in polar coordinates in the neighborhood of $X \in \mathbb{X}$ has form:

$$
d V\left(\exp _{X} t \theta\right)=J(t, \theta) d t d \theta
$$

where, $\quad \theta \in T_{X} \mathbb{X},\|\theta\|=1, t>0, \tilde{t} \in[0, t], \exp _{X} V$ is an exponential map of $\mathrm{V}$ at $\mathrm{X}$ (Appendix $\mathrm{A})$ :

$$
\begin{aligned}
& J(t, \theta)=t^{q-1}+t^{q+1} \operatorname{Ric}_{X}(\theta, \theta)+O\left(t^{q+2}\right) \\
& J(t, \theta)=t^{q-1}+t^{q+1} \operatorname{Ric}_{\tilde{X}}(\tilde{\theta}, \tilde{\theta})
\end{aligned}
$$

$\operatorname{Ric}_{X}(\theta, \theta)$ is a Ricci curvature (26) at $X$, $\tilde{X} \in\left\{\exp _{X} \tilde{t} \theta \mid \tilde{t} \in[0, t]\right\}, \tilde{\theta} \in T_{\tilde{X}}(\mathbb{X})$.

The distance between the closest points of the manifold in q-dimensional space and the distance between them in the q-dimensional Riemannian coordinates Singer and $\mathrm{Wu}$ (2012) are related by Lemma:

Lemma 2. (Coifman and Lafon (2006)). For $X, \tilde{X} \in \mathbb{X}$, such that $\tilde{X}=\exp _{X}(t \theta)$, where $\theta \in T_{X}(\mathbb{X})$ and $\|\theta\|=1$, for small (and small (and small $h=\|X-\tilde{X}\|)$ ):

$$
\begin{aligned}
& t=h+\frac{1}{24}\left\|I I_{X}(\theta, \theta)\right\| h^{3}+O\left(h^{3}\right) \\
& t=h+\frac{1}{24}\left\|I I_{\tilde{X}}(\tilde{\theta}, \tilde{\theta})\right\| h^{3}
\end{aligned}
$$

where, $\quad \tilde{X} \in\left\{\exp _{X} \tilde{t} \theta \mid \tilde{t} \in[0, t]\right\}, \tilde{\theta} \in T_{\tilde{X}}(\mathbb{X}), I_{X}(V, V)$ is a second fundamental form (22).

The values of the right-hand part of the Lemmas 1 and 2 may be bonded under the M1-M8 assumptions:

Lemma 3. For $X \in \mathbb{X}$ and $\theta \in T_{X}(\mathbb{X})$ and $\|\theta\|=1$ :

$$
\left\|I_{X}(\theta, \theta)\right\| \leq C_{I I} \equiv \frac{C_{H}}{c_{J}} \cdot q
$$

where, $C_{H}$ is a maximal element of Hessian matrix (7), $C_{J}$ is a minimum eigenvalue of the metric tensor (5):

Lemma 4. For $X \in \mathbb{X}, \theta \in T_{X}(\mathbb{X})$ and $\|\theta\|=1$ :

$$
\begin{aligned}
& \left\|\operatorname{Ric}_{X}(\theta, \theta)\right\| \leq C_{R i c} \equiv 2 q^{3} \cdot \frac{C_{H}^{2}+C_{T} \sqrt{C_{J}}}{c_{J}^{3 / 2}} \\
& +q^{5} \cdot\left(18 \cdot C_{H}^{2} \frac{C_{J}}{c_{J}}+4 \cdot C_{H} \frac{\sqrt{C_{J}}}{c_{J}}\right) / c_{J}
\end{aligned}
$$

where, $C_{H}$ is a maximum element of Hessian matrix (7), $C_{J}$ and $C_{J}$ are a minimum and maximum eigenvalues of the metric tensor (5) and (6), $C_{T}$ is the maximal norm of the third derivative (8).

The proofs of Lemmas 3 and 4 are in Appendix A.

\section{De Moivre-Laplace Lemmas for Slowly Decreasing Probability}

We use local and integral de Moivre-Laplace lemmas for success parameter which slowly tends to zero. In the classical formulation, the parameter is considered to be fixed. However, the proof almost does not change, if we assume success parameter $p_{n}$ depends on the sample size, but $p_{n} . N \rightarrow \propto$ when $n \rightarrow \propto$. The only difference is the functions expansion in a small parameter $1 /\left(p_{n} \cdot n\right)$, instead of a small parameter $1 / n$ :

Lemma 5. (local de Moivre-Laplace for slowly decreasing probability). Let the success probability in a Bernoulli scheme $p_{n}>0$ depends on the sample size $\mathrm{n}$ and $q_{n}=1-p_{n}$ and also $p_{n} . n \rightarrow \propto$ for $n \rightarrow \propto$. Then for $n$ $\rightarrow \propto$ and the number of successes $k$ such that $\frac{\left|k-n p_{n}\right|}{\left(n p_{n} q_{n}\right)^{2 / 3}} \rightarrow 0$ for $\mathrm{n} \rightarrow \infty$ :

$$
P_{n}(k) \sim \frac{1}{\sqrt{2 \pi n p_{n} q_{n}}} \exp \left(\frac{-\left(k-n p_{n}\right)^{2}}{2 n p_{n} q_{n}}\right)
$$


That is:

$$
\sup _{\left\{k: k-n p_{n} \mid \leq \varphi\left(n p_{n} q_{n}\right)\right\}}\left|\frac{P_{n}(k)}{\frac{1}{\sqrt{2 \pi n p_{n} q_{n}}} \exp \left(\frac{-\left(k-n p_{n}\right)^{2}}{2 n p_{n} q_{n}}\right)}-1\right| \rightarrow 0
$$

where, $\varphi\left(n p_{n} q_{n}\right)$ is an arbitrary function such that $\varphi\left(n p_{n} q_{n}\right)=o\left(\left(n p_{n} q_{n}\right)^{2 / 3}\right)$.

Corollary 2. Lemma is equivalent to the following statement: for each $z \in \mathbb{R}$ such that $z=o\left(n p_{n} q_{n}\right)^{1 / 6}$ and $n p+z \sqrt{n p_{n} q_{n}}$ is integer from $\{1, . ., n\}$ :

$$
p\left(n p+z \sqrt{n p_{n} q_{n}}\right) \sim \frac{1}{\sqrt{2 \pi n p_{n} q_{n}}} e^{-z^{2} / 2}
$$

That is for $\mathrm{n} \rightarrow \propto$ :

$$
\sup _{\{z:|z| \leq \psi(n)\}}\left|\frac{p_{n}\left(n p_{n}+z \sqrt{n p_{n} q}\right)}{\frac{1}{\sqrt{2 \pi n p_{n} q_{n}}} e^{-z^{2} / 2}}-1\right| \rightarrow 0
$$

where, $\psi(n)=o\left(n p_{n} q_{n}\right)^{1 / 6}$.

Lemma 6. (integral de Moivre-Laplace for slowly decreasing probability). Let the success probability in a Bernoulli scheme $p_{n}>0$ depends on the sample size $n$ and $q_{n}=1-p_{n}$ and also $p_{n} . n \rightarrow \infty$ for $n \rightarrow \infty$ Let $\quad P_{n}(k)=C_{n}^{k} p_{n}^{k} q_{n}^{n-k}$, $P_{n}(a, b]=\sum_{a<z \leq b} P_{n}\left(n p_{n}+z \sqrt{n p_{n} q_{n}}\right) . \quad$ Then $\quad \sup _{-\infty \leq a<b \leq \infty}$ $\left|P_{n}(a, b]-\frac{1}{\sqrt{2 \pi}} \int_{a}^{b} \exp \left(-z^{2} / 2\right) d z\right| \rightarrow 0, n \rightarrow \infty$. The proofs of Lemmas 5 and 6 are in Appendix B.

The Probability of Large Deviations for Bounded Random Variables

To estimate the probability of large deviations we will use the following lemma (proved in Appendix B):

Lemma 7. Let $x_{1}, \ldots, x_{n}$ be i.i.d. random variables, $\mathbb{E} \chi_{1}<\infty,\left|\chi_{1}-\mathbb{E} \chi_{1}\right| \leq C<\infty$ and constants $\mathrm{m}_{1}, \ldots, \mathrm{m}_{\mathrm{n}}$ and $\mathrm{m}$, such that $\max _{k=1, \ldots, n}\left|m_{k}-\mathbb{E} \chi_{k}\right| \leq m$. Let $\bar{\chi}=\frac{1}{n} \sum_{k=1}^{n}\left(\chi_{k}-m_{k}\right)$ and $\sigma^{2}=\mathbb{E}\left(\chi_{1}-\mathbb{E} \chi_{1}\right)^{2}$.

Then for $H \geq 2 C$ and $0 \leq x \leq \frac{1}{H}$ :

$$
\begin{aligned}
& P\left(\bar{\chi} \geq x \cdot \sigma^{2}+m\right) \leq \exp \left(-x^{2} \sigma^{2} \cdot n / 4\right) \\
& P\left(\bar{\chi} \leq-x \cdot \sigma^{2}-m\right) \leq \exp \left(-x^{2} \sigma^{2} \cdot n / 4\right)
\end{aligned}
$$

and for $x \geq \frac{1}{H}$ :

$$
\begin{aligned}
& P\left(\bar{\chi} \geq x \cdot \sigma^{2}+m\right) \leq \exp \left(-x \sigma^{2} n / 4 H\right) \\
& P\left(\bar{\chi} \leq-x \cdot \sigma^{2}-m\right) \leq \exp \left(-x \sigma^{2} n / 4 H\right)
\end{aligned}
$$

\section{Integration Area Replacement}

Let $\mathrm{B}_{\varepsilon}(\mathrm{X})$ be the intersection of $\mathrm{p}$-dimensional Euclidian $\varepsilon$-neighborhood of $X \in \mathbb{X}$ and manifold $\mathbb{X}$ :

$$
B_{\varepsilon}(X)=\{\tilde{X} \mid \tilde{X} \in \mathbb{X} \cap\|\tilde{X}-X\|<\varepsilon\}
$$

Let $\tilde{B}_{\varepsilon}(X)$ be the $\varepsilon$-neighborhood of $X$ in locally Riemannian with center $X \in \mathbb{X}$ :

$$
\begin{aligned}
& \tilde{B}_{\varepsilon}(X)=\left\{\tilde{X} \mid \exists V \in T_{X}(\mathbb{X})\right. \\
& \left.\tilde{X}=\exp _{X}(V) \cap\|V\|<\varepsilon\right\}
\end{aligned}
$$

Let $\mathbb{X}_{\varepsilon}$ be the set of internal points which are $\varepsilon$ far from manifold:

$$
\begin{aligned}
& \mathbb{X}_{\varepsilon}=\left\{X \in \mathbb{X} \mid \forall V \in T_{X}(\mathbb{X}) \cap\|V\|<\varepsilon\right. \\
& \left.\exp _{X} V \in \mathbb{X}\right\}
\end{aligned}
$$

Lemma 8. For each bounded function and $g(X, \tilde{X})=\tilde{g}(X, t, \theta), \varepsilon \leq \min \left\{\frac{1}{c(\mathbb{X})}, \sqrt{\frac{24 \cdot(\sqrt[q]{2}-1)}{\max \left\{1, C_{I I}\right\}}}, \frac{1}{2 \sqrt{C_{R i c}}}\right\} \mathrm{a}$ nd $X \in \mathbb{X}_{\varepsilon}, \tilde{X} \in \mathbb{X}, \theta \in T_{X}(\mathbb{X}) \cap\|\theta\|=1$ :

$$
\begin{aligned}
& \left|\int_{B_{\varepsilon}(X)} g(X, \tilde{X}) d V(\tilde{X})-\int_{\tilde{B}_{\varepsilon}(X)} g(X, \tilde{X}) d V(\tilde{X})\right| \\
& \leq 8 \cdot V_{q} \cdot \sup _{X, \tilde{X}}|g(X, \tilde{X})| \cdot \varepsilon^{q+2}
\end{aligned}
$$

where, $c(\mathbb{X})$ is a condition number $(\mathrm{M} 7), V_{q}$ is the volume (14), $C_{I I}$ and $C_{R i c}$ are constants from Lemmas 3 and 4.

Lemma is proved in Appendix B.

\section{Finite Nets}

Additional construction will be used to prove the uniformity of the estimates. $\delta$-net of the metric space $\mathbb{Z}$ is a set $\mathbb{Z}_{\text {net }}(\delta) \subset \mathbb{Z}$ such that for each point $Z \in \mathbb{Z}$ exists $\delta$-close point $Z_{\text {net }} \in \mathbb{Z}_{\text {net }}(\delta)$. 
Since the set of $\mathbb{X}_{\varepsilon}(15)$ far from the border, points is a subset of $\mathbb{X}$ and M2 and M4 hold true, then applying Lemma 10 we get.

Corollary 3. For each $\delta>0$ and $\varepsilon>0$ exist finite $\delta$ net $\mathbb{X}_{\varepsilon}$ with $\left(\frac{2 a \sqrt{p}}{\delta}\right)^{p}$ or fewer elements, where $a>0$ is an edge of p-dimensional hypercube (4).

\section{Proof of Main Theorems}

Let $\chi$ be the indicator the event 'random point $\tilde{X}$, which is distributed with density $p_{\mu}$, is in pdimensional $\varepsilon$-neighborhood of $X \in \mathbb{X}^{\prime}$ :

$$
\chi=\chi(\tilde{X} \mid X, \varepsilon)=I(\|X-\tilde{X}\| \leq \varepsilon)
$$

where, $I(A)=1$, if occurred and else $0 . N_{\varepsilon}(X)(13)$ is a sum of Bernoulli random variables:

$$
N_{\varepsilon}(X)=\sum_{n=1}^{N} \chi\left(X_{1} \mid X, \varepsilon\right)
$$

Let's estimate first and second moments of $\chi$ :

Lemma 9. Let:

$$
\varepsilon \leq \min \left\{\frac{1}{c(\mathbb{X})}, \sqrt{\frac{24 \cdot(\sqrt[q]{2}-1)}{\max \left\{1, C_{I I}\right\}}}, \frac{1}{2 \sqrt{C_{R i c}}}\right\}
$$

$X \in \mathbb{X}_{\varepsilon}$, then $\mathbb{E} \chi^{2}=\mathbb{E} \chi=\varepsilon^{q} \cdot V_{q} \cdot\left(p_{\mu}(X)+\varepsilon^{2} \delta_{\mathbb{E}}\right)$, where:

$$
\begin{aligned}
& \left|\delta_{\mathbb{E}}\right| \leq 8 \cdot p_{\max }+C_{R i c} \\
& +\varepsilon^{2} \cdot\left(\left(C_{R i c} \cdot C_{p, 1}+C_{p, 2}\right)+\varepsilon^{2} \cdot C_{R i c} \cdot C_{p, 2}\right)
\end{aligned}
$$

and constants $V_{q}, p_{\min }, p_{\max }, C_{I I} C_{R i c}, C_{p, 1}$ and $C_{p, 2}$ are defined in (14), (9), (10), Lemmas 3 and 4, (11) and (12). Also, if S3 is not supposed:

$$
\mathbb{E} \chi^{2}=\mathbb{E} \chi \geq \varepsilon^{q} \cdot V_{q} \cdot p_{\text {min }}-V_{q} \cdot \varepsilon^{q+2} \cdot\left(C_{\text {Ric }}+8 p_{\text {max }}\right)
$$

Proof. Note that $\chi^{2}=\chi$. So $\mathbb{E} \chi^{2}=\mathbb{E} \chi$. Let point $\tilde{X} \in \mathbb{X}$ be a random variable with density $p_{\mu}(\tilde{X})$.

$$
\mathbb{E} \chi=\int_{B_{\varepsilon}(X)} p_{\mu}(\tilde{X}) d V(\tilde{X})
$$

Using Lemma 8 for $p_{\mu}(\tilde{X})$ and (10):

$$
\begin{aligned}
& \left|\int_{B_{\varepsilon}(X)} p_{\mu}(\tilde{X}) d V(\tilde{X})-\int_{\tilde{B}_{\varepsilon}(X)} p_{\mu}(\tilde{X}) d V(\tilde{X})\right| \\
& \leq 8 \cdot V_{q} \cdot p_{\max } \cdot \varepsilon^{q+2}
\end{aligned}
$$

Using expansion:

$$
\begin{aligned}
& p_{\mu}(\tilde{X})=p(X)+t \cdot \nabla_{\theta} p(X) \\
& +\frac{t^{2}}{2} \nabla_{\tilde{\theta}} \nabla_{\tilde{\theta}} p(\tilde{X}), \tilde{X} \in \tilde{B}_{\varepsilon}(X)
\end{aligned}
$$

And the symmetry of $\tilde{B}_{\varepsilon}(X)$ :

$$
\begin{aligned}
& \left|\int_{\tilde{B}_{\varepsilon}(X)} p_{\mu}(\tilde{X}) d V(\tilde{X})-\int_{\tilde{B}_{\varepsilon}(X)} p_{\mu}(X) d V(\tilde{X})\right| \\
& =\left|\int_{\tilde{B}_{\varepsilon}(X)}\left(p_{\mu}(\tilde{X})-p_{\mu}(X)\right) d V(\tilde{X})\right| \\
& =\mid \int_{S^{q-1}} \int_{0}^{\varepsilon}\left(t \cdot \nabla_{\theta} p(X)+\frac{t^{2}}{2} \nabla_{\tilde{\theta}} \nabla_{\tilde{\theta}} p(\tilde{X})\right) \\
& \cdot\left(t^{q-1}+t^{q+1} R i c_{\tilde{X}_{2}}(\tilde{\theta}, \tilde{\theta})\right) d t d \theta \mid \\
& \leq\left|\int_{S^{q-1}} \int_{0}^{\varepsilon} t^{q} \cdot \nabla_{\theta} p(X) d t d \theta\right| \\
& +\left|\varepsilon^{4} \cdot\left(C_{R i c} \cdot C_{p, 1}+C_{p, 2}\right) \cdot \int_{S^{q-1}} \int_{0}^{\varepsilon} t^{q-1} d t d \theta\right| \\
& +\left|\varepsilon^{6} \cdot\left(C_{R i c} \cdot C_{p, 2}\right) \cdot \int_{S^{q-1}} \int_{0}^{\varepsilon} t^{q-1} d t d \theta\right| \\
& =0+V_{q} \cdot \varepsilon^{q+4} \cdot\left(\left(C_{R i c} \cdot C_{p, 1}+C_{p, 2}\right)+\varepsilon^{2} \cdot C_{R i c} \cdot C_{p, 2}\right)
\end{aligned}
$$

So:

$$
\begin{aligned}
& \left|\int_{\tilde{B}_{\varepsilon}(X)} p_{\mu}(X) d V(\tilde{X})-V_{q} \cdot \varepsilon^{q} p_{\mu}(X)\right| \\
& =\left|p_{\mu}(X) \cdot \int_{S^{q-1}} \int_{0}^{\varepsilon} t^{q+1} R i c_{\tilde{X}_{2}}(\tilde{\theta}, \tilde{\theta}) d t d \theta\right| \\
& \leq p_{\mu}(X) \cdot \varepsilon^{q+2} \cdot V_{q} \cdot C_{R i c} .
\end{aligned}
$$

and for smooth density (with S3):

$$
\mathbb{E} \chi=\varepsilon^{q} \cdot V_{q} \cdot\left(p_{\mu}(X)+\varepsilon^{2} \delta_{\mathbb{E}}\right)
$$

Where:

$$
\begin{aligned}
& \left|\delta_{\mathbb{E}}\right| \leq 8 \cdot p_{\max }+C_{R i c} \\
& +\varepsilon^{2} \cdot\left(\left(C_{R i c} \cdot C_{p, 1}+C_{p, 2}\right)+\varepsilon^{2} \cdot C_{R i c} \cdot C_{p, 2}\right)
\end{aligned}
$$

Without $\mathrm{S} 3$ for $\mathbb{E} \chi$ :

$$
\begin{aligned}
& \int_{B_{\varepsilon}(X)} p_{\mu}(\tilde{X}) d V(\tilde{X}) \geq \int_{\tilde{B}_{\varepsilon}(X)} p_{\min } d V(\tilde{X}) \\
& -8 \cdot V_{q} \cdot p_{\max } \cdot \varepsilon^{q+2} \geq \varepsilon^{q} \cdot V_{q} \cdot p_{\min }-V_{q} \cdot \varepsilon^{q+2} \cdot\left(C_{R i c}+8 p_{\max }\right)
\end{aligned}
$$

The Lemma is proved.

Corollary 4. If:

$$
\begin{aligned}
& \varepsilon \leq \min \left\{\frac{1}{c(\mathbb{X})}, \sqrt{\frac{24(\sqrt[q]{2}-1)}{\max \left\{1, C_{I I}\right\}}}, \frac{1}{2 \sqrt{C_{R i c}}}\right. \\
& \left.\frac{1}{\sqrt{C_{R i c} C_{p, 2}}}, \frac{1}{\sqrt{C_{R i c} C_{p, 1}+C_{p, 2}+1}}\right\}
\end{aligned}
$$


and $X \in \mathbb{X}_{\varepsilon}$, then in Lemma 8 for $\delta_{\mathbb{E}}(20)$ :

$$
\left|\delta_{\mathbb{E}}\right| \leq C_{\mathbb{E}} \equiv 8 \cdot p_{\max }+C_{R i c}+1 .
$$

Corollary 5. If:

$$
\begin{aligned}
& \varepsilon \leq \min \left\{\frac{1}{c(\mathbb{X})}, \sqrt{\frac{24(\sqrt[q]{2}-1)}{\max \left\{1, C_{I I}\right\}}}, \frac{1}{2 \sqrt{C_{R i c}}}\right. \\
& \left.\frac{1}{\sqrt{C_{R i c} C_{p, 2}}}, \frac{1}{\sqrt{C_{R i c} C_{p, 1}+C_{p, 2}+1}}\right\},
\end{aligned}
$$

and $X \in \mathbb{X}_{\varepsilon}$, then:

$$
\begin{aligned}
& \operatorname{Var} \chi \leq \varepsilon^{q} V_{q} \cdot\left(p_{\mu}(X)+C_{\mathbb{E}} \varepsilon^{2}-p_{\mu}(X)^{2} \varepsilon^{q}+\varepsilon^{q+2} C_{\mathbb{E}}+\varepsilon^{4} C_{\mathbb{E}}^{2}\right) \\
& \operatorname{Var} \chi \geq \varepsilon^{q} V_{q} \cdot\left(p_{\mu}(X)-C_{\mathbb{E}} \varepsilon^{2}-p_{\mu}(X)^{2} \varepsilon^{q}-\varepsilon^{q+2} C_{\mathbb{E}}-\varepsilon^{4} C_{\mathbb{E}}^{2}\right)
\end{aligned}
$$

Proof. Using Lemma 9 and Corollary 4 we transform:

$$
\begin{aligned}
& \operatorname{Var} \chi=\mathbb{E} \chi^{2}-(\mathbb{E} \chi)^{2}=\varepsilon^{q} \cdot V_{q} \cdot\left(p_{\mu}(X)+\varepsilon^{2} \delta_{\mathbb{E}}\right) \\
& \cdot\left(1-\varepsilon^{q} \cdot V_{q} \cdot\left(p_{\mu}(X)+\varepsilon^{2} \delta_{\mathbb{E}}\right)\right) \\
& \leq \varepsilon^{q} V_{q} \cdot\left(p_{\mu}(X)+C_{\mathbb{E}} \varepsilon^{2}-p_{\mu}(X)^{2} \varepsilon^{q}+\varepsilon^{q+2} C_{\mathbb{E}}+\varepsilon^{4} C_{\mathbb{E}}^{2}\right) \\
& \operatorname{Var} \chi=\mathbb{E} \chi^{2}-(\mathbb{E} \chi)^{2} \\
& =\varepsilon^{q} \cdot V_{q} \cdot\left(p_{\mu}(X)+\varepsilon^{2} \delta_{\mathbb{E}}\right) \cdot\left(1-\varepsilon^{q} \cdot V_{q} \cdot\left(p_{\mu}(X)+\varepsilon^{2} \delta_{\mathbb{E}}\right)\right) \\
& \geq \varepsilon^{q} V_{q} \cdot\left(p_{\mu}(X)-C_{\mathbb{E}} \varepsilon^{2}-p_{\mu}(X)^{2} \varepsilon^{q}-\varepsilon^{q+2} C_{\mathbb{E}}-\varepsilon^{4} C_{\mathbb{E}}^{2}\right)
\end{aligned}
$$

The Corollary is proved.

Proof of Theorem 1. Using (19):

$$
\mathbb{E} N_{\varepsilon}(X)=N \cdot \mathbb{E} \chi
$$

As $\mathbb{B}$ is open set (assumption M1), from Corollary 4 for small $\varepsilon$ :

$$
\left|\frac{N_{\varepsilon}(X)}{N}-\varepsilon^{q} V_{q} p_{\mu}(X)\right| \leq \varepsilon^{q+2} V_{q} C_{\mathbb{E}}
$$

Using Chebyshev's inequality for $\frac{N_{\varepsilon}(X)}{N}$ and $\varepsilon^{q} \cdot \psi(N)$,

where

(any $\psi(N)=\left(N \varepsilon^{q}\right)^{-1 / 4} \psi(N): N \varepsilon^{q} \cdot \psi(N)^{2} \rightarrow \infty$ and $\psi(\mathrm{N}) \rightarrow 0$ for $\mathrm{N} \rightarrow 0$ is allowed), we have:

$$
P\left(\left|\frac{N_{\varepsilon}(X)}{N}-\mathbb{E} \chi\right| \geq \varepsilon^{q} \psi(N)\right) \leq \frac{\operatorname{Var} \chi}{N \varepsilon^{2 q} \psi(N)^{2}}
$$

So:

$$
P\left(\left|\frac{N_{\varepsilon}(X)}{N}-\varepsilon^{q} V_{q} p_{\mu}(X)\right| \geq \varepsilon^{q+2} V_{q} C_{\mathbb{E}}+\varepsilon^{q}\right) \leq \frac{\operatorname{Var} \chi}{N \varepsilon^{2 q} \psi(N)^{2}}
$$

Note that for $\mathrm{N} \rightarrow \propto$ :

$$
\begin{aligned}
& \frac{\operatorname{Var} \chi}{N \varepsilon^{2 q} \psi(N)^{2}} \\
& \leq \frac{V_{q} \cdot\left(p_{\mu}(X)+C_{\mathbb{E}} \varepsilon^{2}-p_{\mu}(X)^{2} \varepsilon^{q}+\varepsilon^{q+2} C_{\mathbb{E}}+\varepsilon^{4} C_{\mathbb{E}}^{2}\right)}{N \varepsilon^{q} \psi(N)^{2}} \rightarrow 0 \\
& \varepsilon^{q+2} V_{q} C_{\mathbb{E}}+\psi(N) \rightarrow 0
\end{aligned}
$$

So:

$$
\frac{N_{\varepsilon}(X)}{N \varepsilon^{q} V_{q} p_{\mu}(X)}-1 \stackrel{p}{\longrightarrow} 0 .
$$

The Theorem is proved.

Proof of Theorem 2. As $\mathrm{N} \rightarrow 0$ $I_{\varepsilon}\left(X_{n} \mid X\right)=1 \Rightarrow X_{n} \rightarrow X$.

So:

$$
\begin{aligned}
& p\left(X_{n} \mid I_{\varepsilon}\left(X_{n} \mid X\right)=1\right) \cdot \varepsilon^{q} \\
& =p_{\mu}\left(X_{n}\right) \cdot \frac{\varepsilon^{q}}{P\left(B_{\varepsilon}(X)\right)}=\frac{p_{\mu}\left(X_{n}\right)}{p_{\mu}(X)} \cdot \frac{\varepsilon^{q}}{V_{q} \cdot \varepsilon^{q}} \rightarrow 1 / V_{q}
\end{aligned}
$$

The Theorem is proved.

Proof of Corollary 1. Let be an orthonormal basis in the tangent space $T_{X}(\mathbb{X})$ and $U_{1}, \ldots, U_{p-q}$ be an orthonormal basis in the cotangent space $T_{X}(\mathbb{X})^{\perp}$. Then $V_{1}, \ldots, V_{q}, U_{1}, \ldots, U_{p-q}$ is a basis in $\mathbb{R}^{p}$.

From the Theorems 1 and 2 for the elements of local covariance matrix:

$$
\begin{aligned}
& \frac{\sum_{n=1}^{N} I_{\varepsilon}\left(X_{n} \mid X\right) \cdot\left(\left(X_{n}-X\right)^{T} \cdot V_{i}\right) \cdot\left(\left(X_{n}-X\right)^{T} \cdot V_{j}\right)}{\sum_{n=1}^{N} I_{\varepsilon}\left(X_{n} \mid X\right)} \rightarrow I(i=j) \\
& \frac{\sum_{n=1}^{N} I_{\varepsilon}\left(X_{n} \mid X\right) \cdot\left(\left(X_{n}-X\right)^{T} \cdot V_{i}\right) \cdot\left(\left(X_{n}-X\right)^{T} \cdot U_{j}\right)}{\sum_{n=1}^{N} I_{\varepsilon}\left(X_{n} \mid X\right)} \rightarrow 0 \\
& \frac{\sum_{n=1}^{N} I_{\varepsilon}\left(X_{n} \mid X\right) \cdot\left(\left(X_{n}-X\right)^{T} \cdot U_{i}\right) \cdot\left(\left(X_{n}-X\right)^{T} \cdot U_{j}\right)}{\sum_{n=1}^{N} I_{\varepsilon}\left(X_{n} \mid X\right)} \rightarrow 0
\end{aligned}
$$

The Corollary is proved.

Proof of Theorem 3. $\chi$ (18) has Bernoulli distribution, so using Lemma 8 and it's Corollaries we get the conditions of Lemma 6 for $\chi$ : 
$\mathbb{E} \chi \rightarrow 0$ and $N \cdot \mathbb{E} \chi \rightarrow \infty$ for $N \rightarrow \propto$. It remains to note that for $N \rightarrow \propto$ :

$$
\begin{aligned}
& \frac{\mathbb{E} \chi}{\varepsilon^{q} V_{q} p_{\mu(X)}} \rightarrow 1 \\
& \frac{\operatorname{Var} \chi}{\varepsilon^{q} V_{q} p_{\mu(X)}} \rightarrow 1
\end{aligned}
$$

So by Lemma 8:

$$
\frac{N_{\varepsilon}(X)-N \varepsilon^{q} p_{\mu}(X) V_{q}}{\sqrt{N \varepsilon^{q} p_{\mu}(X) V_{q}}} \stackrel{d}{\longrightarrow} N(0,1)
$$

The Theorem is proved.

Proof of Theorem 4 . We verify the conditions of Lemma 7 for $\chi(18), m_{k}=\varepsilon^{q} . V_{p} \cdot p_{\mu}(X), m=\varepsilon^{q+2} C_{\mathbb{E}}$ (Corollary 4) and:

$$
\begin{aligned}
& |\chi-\mathbb{E} \chi| \leq 1 \\
& \left|m_{k}-\mathbb{E} \chi_{k}\right| \leq \varepsilon^{q+2} C_{\mathbb{E}}
\end{aligned}
$$

$$
\begin{aligned}
& \text { For } \varepsilon<\min \left\{\frac{1}{\sqrt{4 C_{\mathbb{E}} p_{\min }}}, \sqrt[q]{\frac{1}{4 p_{\min }}}\right\} \text { by Corollary 4: } \\
& \operatorname{Var} \chi \leq \varepsilon^{q} V_{q} \\
& \cdot\left(p_{\mu}(X)+C_{\mathbb{E}} \varepsilon^{2}-p_{\mu}(X)^{2} \varepsilon^{q}+\varepsilon^{q+2} C_{\mathbb{E}}+\varepsilon^{4} C_{\mathbb{E}}^{2}\right) \\
& \leq \varepsilon^{q} V_{q} \cdot p_{\mu}(X)(1+1 / 4+1 / 4+1 / 4)<2 \varepsilon^{q} V_{q} \cdot p_{\mu}(X) \\
& \operatorname{Var} \chi \geq 1 / 4 \cdot \varepsilon^{q} V_{q} \cdot p_{\mu}(X)
\end{aligned}
$$

Thus, the conditions of Lemma 7 fulfilled and for $z$ $\leq 1 / 16$ :

$$
\begin{aligned}
& P\left(\frac{N_{\varepsilon}(X)}{N \varepsilon^{q} V_{q} p_{\mu}(X)}-1 \geq z+\varepsilon^{2} \cdot \frac{C_{\mathbb{E}}}{V_{q} p_{\mu}}\right) \\
& \leq \exp \left(-z^{2} \cdot N \varepsilon^{q} V_{q} p_{\mu}(X) / 4\right) \\
& P\left(\frac{N_{\varepsilon}(X)}{N \varepsilon^{q} V_{q} p_{\mu}(X)}-1 \leq-z-\varepsilon^{2} \cdot \frac{C_{\mathbb{E}}}{V_{q} p_{\mu}}\right) \\
& \leq \exp \left(-z^{2} \cdot N \varepsilon^{q} V_{q} p_{\mu}(X) / 4\right)
\end{aligned}
$$

Similarly, if the S3 is not fulfilled, then replacing the evaluation density expansion in Taylor's formula at their rough equivalents.

The Theorem is proved.

Proof of Theorem 5. Indicator function is discontinuous, so $N_{\varepsilon}(X)$ is a discontinuous function too. However, due to nesting balls $\tilde{B}_{\varepsilon / 3}(X) \subset \tilde{B}_{\varepsilon}(\tilde{X})$ for $X \in \tilde{B}_{\varepsilon / 3}(\tilde{X})$ and $\varepsilon$ :

$$
N_{\varepsilon}(\tilde{X}) \geq N_{\varepsilon / 3}(X)
$$

So we get the statement of this Theorem by Corollary 3, Lemma 11 and Theorem 3.

The Theorem is proved.

\section{Conclusion}

Random variable 'the number of points in the neighborhood of a fixed point on manifold' is considered in the present paper. Points are assumed to lie on a good enough unknown manifold, the neighborhood is ball shaped and Euclidean, its radius slowly tends to zero with sample size growth.

Asymptotic expansion and uniform large deviation results are obtained for the considered random variable. The problem statement is motivated by manifold learning problems (Roweis and Saul, 2000; Zhang and Zha, 2004; Bernstein and Kuleshov, 2014).

The results of the paper could be used for the manifold learning algorithms analysis and could be generalized to get asymptotic properties of all algorithm steps.

\section{Acknowledgement}

The study was performed in the IITP RAS exclusively by the grant from the Russian Science Foundation (project № 14-50-00150).

\section{Ethics}

The author hereby declares no conflict of interest with regard to this manuscript.

\section{Appendix A. Definitions and Lemmas from Differential Geometry}

In this Section, we introduce the necessary information for further consideration related to the differential geometry.

\section{Covariant Derivative}

Differentiation on manifolds refers as usual. We recall that does it mean. It is easy to determine the derivative of a function in a given direction $V \in \mathbb{R}^{p} \backslash\{0\}$ at a given point $Z \in \mathbb{R}^{p}$ of the p-dimensional real space $\mathbb{R}^{p}$. However, for $X \in \mathbb{X}$ even a small displacement in all directions leaves the transfer result from the manifold. More precisely, for the manifold $\mathbb{X}$ covered with the map $(\mathbb{B}, f)$ and full rank Jacobi matrix $J_{F}\left(f^{-1}(X)\right)$, at $X_{0}=f\left(b_{0}\right)$ manifold locally behaves almost like a q-dimensional linear space tangent space $T_{X}(\mathbb{X})$, which can be defined as a linear space spanned by the p-dimensional column vectors of the matrix: 


$$
J_{f}\left(b_{0}\right)=\left(\frac{\partial f}{\partial b^{1}}\left(b_{0}\right)|\ldots| \frac{\partial f}{\partial b^{q}}\left(b_{0}\right)\right)
$$

where, the superscript denotes the component of the vector. That is, one can only differentiate in directions $V\left(X_{0}\right) \in T_{X_{0}}(\mathbb{X})$. Therefore, the tangent space $T_{X_{0}}(\mathbb{X})$ depends on the point $\mathrm{X}_{0}$ of the manifold, in which it is defined. Hence, on the manifold one cannot determine the derivative of a scalar function $\varphi(X)$ as the limit of changes in the function $\varphi$ from point $X_{0}$ to point $X_{0}+t V\left(X_{0}\right)$ over the length of the $t V\left(X_{0}\right)$, since $X_{0}+t V\left(X_{0}\right) \notin \mathbb{X}$ and the value of $\varphi$ at this point is not defined in the general case. So, instead of $X_{0}+T v\left(X_{0}\right)$ a curve $\gamma(\mathrm{t}), \mathrm{t} \in(-\varepsilon, \varepsilon)$ is considered on the manifold, such that $\gamma(0)=X_{0}$ and $\frac{\partial \gamma}{\partial t}(0)=V\left(X_{0}\right)$. The derivative of a scalar function is defined as $\nabla_{V\left(X_{0}\right)} \varphi\left(X_{0}\right)=\lim _{t \rightarrow 0} \frac{\varphi(\gamma(t))-\varphi(\gamma(0))}{t}=\lim _{t \rightarrow 0} \frac{\varphi(\gamma(t))-\varphi\left(X_{0}\right)}{t}$.

Note. The identity covering exists in the case of Euclidean space $\mathbb{R}^{m}: \mathbb{B}=\mathbb{R}^{m}$ and $f(b)=b$, i.e., $X=b$. Therefore, as a curve $\gamma(t)$, one can choose $\gamma(t)=X_{0}+t V\left(X_{0}\right)$ and the covariant derivative of the function $\varphi$ coincides with the usual directional derivative.

Consider the restriction of the vector field $V(X) \in T_{X}(\mathbb{X}), X \in \mathbb{X} \quad$ on the curve $\gamma(\mathrm{t})$ : $\tilde{V}(t)=V(\gamma(t)), t \in(-\varepsilon, \varepsilon)$. The derivative of $\tilde{V}(t)$ is defined as usual:

$$
\frac{\partial \tilde{V}}{\partial t}(t)=\lim _{h \rightarrow 0} \frac{\tilde{V}(t+h)-\tilde{V}(t)}{t}
$$

where, $\mathrm{t} \in(-\varepsilon, \varepsilon)$. However, the derivative $\frac{\partial \tilde{V}}{\partial t}(t)$ can be not in the $T_{\gamma(t)}(\mathbb{X})$. Therefore, one can define the covariant derivative $\frac{D \tilde{V}}{d t}(h)$ as a projection $\frac{\partial \tilde{V}}{\partial t}(t)$ on $T_{\gamma(t)}(\mathbb{X})$.

Next, we consider the equation:

$$
\left\{\begin{array}{l}
\frac{D \tilde{W}}{d t}(t)=0 \\
\tilde{W}(0)=\tilde{W}
\end{array}\right.
$$

where, $W \in T_{\gamma(0)}(\mathbb{X})$. It's solution $\tilde{W}(t)$ exist and is called parallel transport of $\tilde{W}(t)$ over $\gamma(\mathrm{t})$ and is designated as $\tilde{W}(t)=P_{\gamma(t), \gamma(0)} \tilde{W}$. Now, to determine the derivative of the vector field $W(X), X \in \mathbb{X}$ along a curve $\gamma(\mathrm{t}), \mathrm{t} \in(-\varepsilon, \varepsilon)$. We will transfer $\mathrm{W}(\gamma(\mathrm{t}))$ from point $X_{0}=\gamma(0)$. The result of the parallel transport $P_{\gamma(0), \gamma(t)} W(\gamma(t)) \in T_{\gamma(0)}(\mathbb{X})$, so the difference $P_{\gamma(0), \gamma(t)} W(\gamma(t))-W(\gamma(0))$ is determined in $T_{\gamma(0)}(\mathbb{X})$ and the covariant derivative is defined for the vector field $W(X)$ on $\mathbb{X}$ :

$$
\nabla_{V\left(X_{0}\right)} W\left(X_{0}\right)=\lim _{t \rightarrow 0} \frac{P_{\gamma(0), \gamma(t)} W(\gamma(t))-W(\gamma(0))}{t}
$$

where, $\quad \gamma:(-\varepsilon, \varepsilon) \rightarrow \mathbb{X} \quad$ and $\quad \gamma(0)=X_{0} \in \mathbb{X}$, $\gamma^{\prime}(0)=V\left(X_{0}\right) \in T_{X_{0}}(\mathbb{X})$.

$$
\begin{aligned}
& \text { Note. For the Euclidian space } \mathbb{R}^{m} \text { the } \\
& \text { Solution } \quad(21) \quad \text { is } \\
& P_{\gamma(0), \gamma(t)} W(\gamma(t))=W(\gamma(t))=W\left(X_{0}+t V\left(X_{0}\right)\right) \text { and }
\end{aligned}
$$
covariant derivative of the vector field equals to directional derivative:

$$
\nabla_{V\left(X_{0}\right)} W\left(X_{0}\right)=\lim _{t \rightarrow 0} \frac{W\left(X_{0}+t V\left(X_{0}\right)\right)-W\left(X_{0}\right)}{t}
$$

We will denote the covariant derivative in the direction $V \in T_{X}(\mathbb{X}) \backslash\{0\}$ at $X$ as $\nabla_{V}$. The covariant derivative has the usual properties of the directional derivative, for example, linearity $V_{1}, V_{2} \in T_{X}(\mathbb{X}) \backslash\{0\}$, $\alpha_{1}, \alpha_{2} \in \mathbb{R} \backslash 0$ for $\alpha_{1} V_{1}+\alpha_{2} V_{2} \neq 0$ :

$$
\nabla_{\alpha_{1} V_{1}+\alpha_{2} V_{2}}=\alpha_{1} \nabla_{V_{1}}+\alpha_{2} \nabla_{V_{2}}
$$

\section{Quadratic Forms and Ricci Curvature}

In this study, it is assumed that the metric tensor of the manifold $\mathbb{X}$ is generated by embedding at multidimensional space $\mathbb{R}^{p}$. This means that the scalar product of vectors $V, W \in T_{X}(\mathbb{X})$ is the restriction of the scalar product of $\mathbb{R}^{p}$ on $T_{X}(\mathbb{X})$, that is, in an orthonormal basis is expressed by a bilinear form $I_{X}(V, W)=V^{T} W$. The first quadratic form of manifold is the length of the vectors of the tangent space and allows to calculate the lengths of curves:

$$
I_{X}(V, V)=V^{T} V .
$$

We write down the coordinates of the vector in the basis induced by parameterization $(\mathbb{B}, f)$ :

$$
\begin{aligned}
& b=f^{-1}(X) \\
& V=J_{f}(b) \alpha_{V} \\
& \alpha_{V} \in \mathbb{R}^{q}, \alpha_{V}=\left(J_{f}(b)^{T} J_{f}(b)\right)^{-1} J_{f}(b)^{T} V \\
& I_{X}^{c}\left(\alpha_{v}\right)=\alpha_{v}^{T}\left(J_{f}(b)^{T} J_{f}(b)\right) \alpha_{v}
\end{aligned}
$$


The second bilinear form shows the orthogonal to the tangent space component change of vectors from the tangent space along different directions $V, W \in T_{X}(\mathbb{X})$. We write the expression of the second bilinear form $I I_{X}(V, W)$ in the basis, given parameterization $(\mathbb{B}, f)$ and point $X=f(b)$ :

$$
I I_{X}(V, W)=\pi(X)^{\perp} \cdot \sum_{i, j=1}^{q} \frac{\partial^{2} f(b)}{\partial b_{i} \partial b_{j}} \alpha_{V, i} \alpha_{W, j}
$$

where, $\alpha_{V}=\left(\alpha_{V, 1}, \ldots, \alpha_{V, q}\right)^{T}$ and $\alpha_{W}=\left(\alpha_{W, 1}, \ldots, \alpha_{W, q}\right)^{T}$ are coordinates of $V$ and $W$ in the parametric basis, $\frac{\partial^{2} f(b)}{\partial b_{i} \partial b_{j}} \in \mathbb{R}^{p}, \pi(X)^{\perp}=\left(I-J_{f}(b)\left(J_{f}^{T}(b) J_{f}(b)\right)^{-1} J_{f}(b)^{T}\right)$ is a projector on cotangent space $\left(T_{X}(\mathbb{X})\right)^{\perp}$.

The second fundamental form of $I I_{X}(V, V)$ determines the normal curvature of the manifold.

To determine the Ricci curvature we introduce the notation for the Christoffel symbols. Define:

$g_{i j}=\frac{\partial f(b)}{\partial b_{i}} \Gamma_{j k, l}=\frac{1}{2}\left(\frac{\partial g_{j l}}{\partial b_{k}}+\frac{\partial g_{k l}}{\partial b_{j}}-\frac{\partial g_{j k}}{\partial b_{l}}\right)$

$\Gamma_{j k, l}$ are called Christoffel symbols of the first kind. Let $g^{i j}$ be elements of the inverse matrix $J_{F}^{T}(b) J_{F}(b)$, i.e. the elements of the matrix $\left(J_{f}^{T}(b) J_{f}(b)\right)^{-1}$. The Christoffel symbols of the second kind:

$$
\Gamma_{j k}^{i}=\sum_{l=1}^{q} \Gamma_{j k, l} \cdot g^{l i}
$$

Elements of the Ricci curvature tensor are defined as:

$$
\begin{aligned}
R_{i j} & =\sum_{k=1}^{q}\left(\frac{\partial \Gamma_{j i}^{k}}{\partial b_{k}}-\frac{\partial \Gamma_{k i}^{k}}{\partial b_{j}}+\sum_{l=1}^{q} \Gamma_{k l}^{k} \Gamma_{j i}^{l}-\Gamma_{j i}^{k} \Gamma_{k i}^{l}\right) \\
i, j & =1, \ldots, q
\end{aligned}
$$

Let $R=\left(R_{i j}\right)_{i, j=1}^{q}$ be the matrix of rank q. For $\theta \in T_{X}(\mathbb{X}): \quad \alpha_{\theta}=\left(J_{f}(b)^{T} J_{f}(b)\right)^{-1} J_{f}(b)^{T} \theta$. Ricci curvature in the direction of $\theta$ at $X$ :

$$
\operatorname{Ric}_{X}(\theta, \theta)=\alpha_{\theta}^{T} \cdot R \cdot \alpha_{\theta}
$$

Ricci curvature describes the difference between Euclidean volume element and the manifold volume element.

\section{Locally Riemannian Coordinates and Exponential Map}

For a small neighborhood of $X_{0} \in \mathbb{X}$ mapping $X \mapsto V \in T_{X}(\mathbb{X}): \quad X=f(\gamma(1))$, where $\gamma: \gamma(0)=X_{0}$ and $\frac{\partial \gamma}{\partial t}(0)=V$, defines a one-to-one correspondence between the neighborhood of $X_{0}$ and the neighborhood of 0 in $T_{X_{0}}(\mathbb{X})$. The inverse mapping is called exponential and is denoted $X=\exp _{X_{0}}(V)$.

In this neighborhood vectors $V \in T_{X_{0}}(\mathbb{X})$ are coordinates. These coordinates are called locally Riemann coordinates.

$$
\begin{aligned}
& \text { Note. For the Euclidian space } \mathbb{X}=\mathbb{R}^{m} \text { the } \\
& \text { tangent space is } T_{X}(\mathbb{X})=\mathbb{R}^{m} \text { and } \\
& \exp _{X_{0}}(V)=X_{0}+V \text { for each } V \in T_{X}(\mathbb{X}) \text { (that } \\
& \text { is, the result is valid for an arbitrary } \\
& \text { neighborhood of } \left.X_{0}\right) \text {. }
\end{aligned}
$$

The distance between the points of the manifold in pdimensional space and the distance between them in the qdimensional Riemannian coordinates are related with Lemmas 1 and 2. Lemmas 3 and 4 roughly upper bound the second fundamental form and Ricci curvature through a fairly smooth parameterization of the manifold.

We prove these Lemmas:

Proof of Lemma 3. For an arbitrary point $b \in \mathbb{B}$ denote by $V_{1}, \ldots, V_{q}$ orthonormal basis of eigenvectors of $J_{f}(b)^{T} J_{f}(b)$. Let $\lambda_{1}, \ldots, \lambda_{q}$ be the corresponding to $V_{1}, \ldots, V_{q}$ eigenvalues. Let $\theta=\sum_{i=1}^{q} \beta_{i} V_{i}$, where $\sum_{i=1}^{q} \beta_{i}^{2}=1$, be the coordinates $\theta \in T_{f(b)}(\mathbb{X}):\|\theta\|=1$ is the basis $V_{1}, \ldots, V_{q}$. Using (5):

$$
\begin{aligned}
& \alpha_{\theta}=\left(J_{f}(b)^{T} J_{f}(b)\right)^{-1} J_{f}(b)^{T} \theta \\
& =\left(J_{f}(b)^{T} J_{f}(b)\right)^{-1} J_{f}(b)^{T} \sum_{i=1}^{q} \beta_{i} V_{i} ; \\
& \left\|\alpha_{\theta}\right\|^{2}=\sum_{i=1}^{q} \frac{1}{\lambda_{i}} \beta_{i}^{2} ;\left\|\alpha_{\theta}\right\|^{2} \leq \frac{1}{c_{J}} .
\end{aligned}
$$

$$
\text { Using } X=f(b)(7),(27) \text { : }
$$

$$
\begin{aligned}
& \left\|\mathrm{II}_{X}(\theta, \theta)\right\|=\left\|\pi^{\perp}(X) \cdot \sum_{i, j=1}^{q} \frac{\partial^{2} f(b)}{\partial b_{i} \partial b_{j}} \alpha_{\theta, i} \alpha_{\theta, j}\right\| \\
& \leq\left\|\sum_{i, j=1}^{q} \frac{\partial^{2} f(b)}{\partial b_{i} \partial b_{j}} \alpha_{\theta, i} \alpha_{\theta, j}\right\| \leq C_{H} \cdot\left\|\alpha_{\theta}\right\|_{1}^{2} \leq C_{H} \cdot q \cdot \frac{1}{c_{J}}
\end{aligned}
$$


The Lemma is proved.

Proof of Lemma 4. Using (23) and (24) we estimate (25) by triangle inequality:

$$
\begin{aligned}
& R_{i j}|=| \sum_{k=1}^{q}\left(\frac{\partial \Gamma_{j i}^{k}}{\partial b_{k}}-\frac{\partial \Gamma_{k i}^{k}}{\partial b_{j}}+\sum_{l=1}^{q} \Gamma_{k l}^{k} \Gamma_{j i}^{l}-\Gamma_{j l}^{k} \Gamma_{k i}^{l}\right) \mid X_{1}, \ldots, X_{n} \\
& \leq 2 q \cdot \max _{i, j, k=1, \ldots, q}\left|\frac{\partial \Gamma_{j i}^{k}}{\partial b_{k}}\right|+2 q^{2} \cdot\left(\max _{j, i, k=1, \ldots, q}\left|\Gamma_{j i}^{l}\right|\right)^{2} \\
& \left|\Gamma_{j k}^{i}\right|=\left|\sum_{l=1}^{q} \Gamma_{j k, l} g^{l i}\right|=\left|\sum_{l=1}^{q} \frac{1}{2}\left(\frac{\partial g_{j l}}{\partial b_{k}}+\frac{\partial g_{k l}}{\partial b_{j}}-\frac{\partial g_{j k}}{\partial b_{l}}\right) g^{l i}\right| \\
& \leq \frac{3 q}{2} \cdot \max _{k, l, j=1, \ldots, q}\left|\frac{\partial g_{j l}}{\partial b_{k}}\right| \cdot \max _{k, l=1, \ldots, q}\left|g^{k l}\right| \\
& \left|\frac{\partial g_{j l}}{\partial b_{k}}\right|=\left|\frac{\partial\left(\frac{\partial f(b)}{\partial b_{j}}, \frac{\partial f(b)}{\partial b_{l}}\right)}{\partial b_{k}}\right| \\
& \leq 2 \cdot \max _{j, k=1, \ldots, q}\left\|\frac{\partial^{2} f(b)}{\partial b_{j} \partial b_{k}}\right\| \cdot \max _{j=1, \ldots, q}\left\|\frac{\partial f(b)}{\partial b_{j}}\right\| \\
& \leq 2 \cdot C_{H} \cdot \sqrt{C_{J}} ; \max _{k, l=1, \ldots, q}\left|g^{k l}\right| \leq 1 / \sqrt{c_{J}} \\
& \left|\frac{\partial \Gamma_{j i}^{k}}{\partial b_{k}}\right|=\left|\sum_{l=1}^{q} \frac{\partial \Gamma_{j k, l}}{\partial b_{k}} g^{l i}-\sum_{l_{1}, i_{1}=1}^{q} \Gamma_{j k, l} \cdot g^{l l_{1}} \frac{\partial g_{l_{1} i_{1}}}{\partial b_{k}} g^{i_{i} i}\right| \\
& \leq q \cdot \max _{j, k, l=1, \ldots, q}\left|\frac{\partial \Gamma_{j k, l}}{\partial b_{k}}\right| \cdot \max _{k, l=1, \ldots, q}\left|g^{k l}\right| \\
& +q^{3} \cdot \max _{j, k, l=1, \ldots, q}\left|\Gamma_{j k, l}\right| \cdot\left(\max _{k, l=1, \ldots, q}\left|g^{k l}\right|\right)^{2} \\
& \max _{j, l, k=1, \ldots, q}\left|\frac{\partial g_{j l}}{\partial b_{k}}\right| \\
& \leq q / \sqrt{c_{J}} \cdot \max _{j, k, l=1, \ldots, q}\left|\frac{\partial \Gamma_{j k, l}}{\partial b_{k}}\right|+2 q^{3} \cdot C_{H} \cdot \sqrt{C_{J}} / c_{J} \\
& \left|\frac{\partial \Gamma_{j k, l}}{\partial b_{k}}\right| \leq 3 / 2 \cdot \max _{i, j, k, m=1, \ldots, q}\left|\frac{\partial^{2}\left(\frac{\partial f(b)}{\partial b_{j}}, \frac{\partial f(b)}{\partial b_{l}}\right)}{\partial b_{k} \partial b_{m}}\right| \\
& \leq 3 \cdot \max _{i, j, k, m=1, \ldots, q}\left|\frac{\partial\left(\frac{\partial^{2} f(b)}{\partial b_{j} \partial b_{k}}, \frac{\partial f(b)}{\partial b_{l}}\right)}{\partial b_{m}}\right| \leq 3 \cdot\left(C_{H}^{2}+C_{T} \cdot \sqrt{C_{J}}\right)
\end{aligned}
$$

So:

$$
\left|R_{i j}\right| \leq 2 q^{2} \cdot \frac{C_{H}^{2}+C_{T} \sqrt{C_{J}}}{\sqrt{c_{J}}}+q^{4} \cdot\left(18 \cdot C_{H}^{2} \frac{C_{J}}{c_{J}}+4 \cdot C_{H} \frac{\sqrt{C_{J}}}{c_{J}}\right)
$$

Using (26) and (27):

$$
\begin{aligned}
& \left\|\operatorname{Ric}_{X}(\theta, \theta)\right\|=\left\|\alpha_{\theta}^{T} \cdot R \cdot \alpha_{\theta}\right\| \leq \max _{i, j=1, \ldots, q}\left|R_{i j}\right| \cdot\left\|\alpha_{\theta}\right\|_{1}^{2} \\
& \leq 2 q^{3} \cdot \frac{C_{H}^{2}+C_{T} \sqrt{C_{J}}}{c_{J}^{3 / 2}}+q^{5} \cdot\left(18 \cdot C_{H}^{2} \frac{C_{J}}{c_{J}}+4 \cdot C_{H} \frac{\sqrt{C_{J}}}{c_{J}}\right) / c_{J}
\end{aligned}
$$

The Lemma is proved.

\section{Appendix B. Lemmas Proofs}

In this section, Lemmas used in the proof of the main theorems are listed and proved.

\section{De Moivre-Laplace for Slowly Decreasing Success Probability}

We prove local and integral de Moivre-Laplace lemmas for slowly tending to zero success parameter.

Proof of Lemma 5. The proof almost repeats the proof of the local limit theorem from Shiryayev (1984) and essentially uses the Stirling formula $n !=\sqrt{2 \pi n} \cdot e^{-n} n^{n} \cdot(1+R(n))$,

where

$R(n)=\frac{1}{12 n}+\frac{1}{288 n^{2}}-\frac{139}{51840 n^{3}}+O\left(\frac{1}{n^{4}}\right)$ and $\frac{1}{12 n}>R(n)>\frac{1}{13 n}, n \geq 1$ could be written via Bernoulli numbers. We will omit subscript $\mathrm{n}$ in $p_{n}$ и $q_{n}$ for convenience.

For $n \rightarrow \infty, k \rightarrow \infty, n-k \rightarrow \infty$ :

$$
\begin{aligned}
& C_{n}^{k}=\frac{n !}{k !(n-k) !} \\
& =\frac{\sqrt{2 \pi n} \cdot e^{-n} n^{n}}{\sqrt{2 \pi k \cdot 2 \pi(n-k)} \cdot e^{-k} k^{k} \cdot e^{-(n-k)}(n-k)^{n-k}} \\
& =\frac{1}{\sqrt{2 \pi n \frac{k}{n} \cdot\left(1-\frac{k}{n}\right)}} \cdot \frac{1+r}{\left(\frac{k}{n}\right)^{k}\left(1-\frac{k}{n}\right)^{n-k}}
\end{aligned}
$$

Where:

$$
r=r(n, k, n-k)=\frac{1+R(n)}{(1+R(k))(1+R(n-k))}
$$

Estimate $r$ for $k>1, n-k>1$ :

$$
\begin{aligned}
& 1>\frac{1+R(n)}{(1+R(k))(1+R(n-k))}>\left(1+\frac{1}{13 n}\right)\left(1-\frac{1}{12 k}\right) \\
& \left(1-\frac{1}{12(n-k)}\right)>1-\frac{1}{12 k}-\frac{1}{12(n-k)} \\
& |r| \leq \frac{1}{12 k}+\frac{1}{12(n-k)}
\end{aligned}
$$

So: 


$$
p(k)=C_{n}^{k} p^{k} q^{n-k}=\frac{1}{\sqrt{2 \pi n \frac{k}{n}\left(1-\frac{k}{n}\right)}} \cdot \frac{p^{k}(1-p)^{n-k}}{\left(\frac{k}{n}\right)^{k}\left(1-\frac{k}{n}\right)^{n-k}} \cdot(1+r)
$$

Denote $\hat{p}=\frac{k}{n}, \hat{q}=1-\hat{p} . \quad p-\hat{p}=\hat{q}-q$ because of $p+q=\hat{p}+\hat{q}=1$. Also, $\frac{p-\hat{p}}{p}$ and $\frac{q-\hat{q}}{q}=-\frac{p-\hat{p}}{q}$ are small parameters as $\frac{p-\hat{p}}{p q}=\frac{n p-k}{n p q}$ is small from the lemmas assumptions and $0<p, q<1$. So:

$$
\begin{aligned}
& p(k)=\frac{1}{\sqrt{2 \pi n \hat{p}(1-\hat{p})}}\left(\frac{p}{\hat{p}}\right)^{k}\left(\frac{1-p}{1-\hat{p}}\right)^{n-k} \cdot(1+r) \\
& =\frac{1}{\sqrt{2 \pi n \hat{p}(1-\hat{p})}} \exp \left(k \ln \frac{p}{\hat{p}}+(n-k) \ln \frac{1-p}{1-\hat{p}}\right)^{n-k} \cdot(1+r) \\
& =\frac{1}{\sqrt{2 \pi n \hat{p}(1-\hat{p})}} \exp \left(n \cdot\left(\hat{p} \ln \frac{p}{\hat{p}}+\hat{q} \ln \frac{q}{\hat{q}}\right)\right) \cdot(1+r) \\
& =\frac{1}{\sqrt{2 \pi n \hat{p}(1-\hat{p})}} \exp \left(-n \cdot\left(p \cdot\left(1+\frac{\hat{p}-p}{p}\right) \ln \left(1+\frac{\hat{p}-p}{p}\right)\right.\right. \\
& \left.\left.+q \cdot\left(1-\frac{\hat{p}-p}{q}\right) \ln \left(1-\frac{\hat{p}-p}{q}\right)\right)\right) \cdot(1+r)
\end{aligned}
$$

We use an expansion in Taylor's formula with the remainder term in the Lagrange form:

$$
\begin{aligned}
& \left(1+\frac{\hat{p}-p}{p}\right) \ln \left(1+\frac{\hat{p}-p}{p}\right)=-\frac{\hat{p}-p}{p}+\frac{1}{2} \cdot\left(\frac{\hat{p}-p}{p}\right)^{2} \\
& +\left(\frac{1}{2}-\frac{1}{3} \cdot \frac{1}{\left(1+\delta_{p}\right)^{3}}\right) \cdot\left(\frac{\hat{p}-p}{p}\right)^{2} \\
& \left(1-\frac{\hat{p}-p}{q}\right) \ln \left(1-\frac{\hat{p}-p}{q}\right)=\frac{\hat{p}-p}{q}+\frac{1}{2} \cdot\left(\frac{\hat{p}-p}{q}\right)^{2} \\
& +\left(-\frac{1}{2}+\frac{1}{3} \cdot \frac{1}{\left(1-\delta_{q}\right)^{3}}\right) \cdot\left(\frac{\hat{p}-p}{q}\right)^{2} \\
& p\left(1+\frac{\hat{p}-p}{p}\right) \ln \left(1+\frac{\hat{p}-p}{p}\right)+q\left(1-\frac{\hat{p}-p}{q}\right) \ln \left(1-\frac{\hat{p}-p}{q}\right) \\
& =\frac{1}{2}\left(\frac{1}{p}+\frac{1}{q}\right)(\hat{p}-p)^{2}+\left(\frac{1}{2}-\frac{1}{3} \cdot \frac{1}{\left(1+\delta_{p}\right)^{2}}\right) \cdot \frac{(\hat{p}-p)^{3}}{p^{2}} \\
& +\left(-\frac{1}{2}+\frac{1}{3} \cdot \frac{1}{\left(1-\delta_{q}\right)^{2}}\right) \cdot \frac{(\hat{p}-p)^{3}}{q^{2}}
\end{aligned}
$$

where, $\quad \delta_{p} \in\left[\min \left\{0, \frac{\hat{p}-p}{p}\right\}, \max \left\{0, \frac{\hat{p}-p}{p}\right\}\right] \quad$ and $\delta_{q} \in\left[\min \left\{0, \frac{\hat{p}-p}{q}\right\}, \max \left\{0, \frac{\hat{p}-p}{q}\right\}\right]$.

Also

$\frac{1}{p}+\frac{1}{q}=\frac{p+q}{p q}=\frac{1}{p q}$

and

$\frac{n}{2}\left(\frac{1}{p}+\frac{1}{q}\right)(\hat{p}-p)^{2}=\frac{n}{2 p q}\left(\frac{k}{n}-p\right)^{2}=\frac{(k-n p)^{2}}{2 n p q}$.

So:

$$
\frac{n}{2 p q}\left(\frac{k}{n}-p\right)^{2}=\frac{(k-n p)^{2}}{2 n p q}
$$

We get:

$$
p(k)=\frac{1}{\sqrt{2 \pi n p q}} \exp \left(-\frac{(k-n p)^{2}}{2 n p q}\right) \cdot(1+\tilde{r}(n, k, n-k))
$$

Where:

$$
\begin{aligned}
& 1+\tilde{r}(n, k, n-k)=(1+r(n, k, n-k)) \\
& \cdot \exp \left(-n \cdot\left(\left(\frac{1}{2}-\frac{1}{3} \cdot \frac{1}{\left(1+\delta_{p}\right)^{2}}\right)\right.\right. \\
& \left.\left.\cdot \frac{(\hat{p}-p)^{3}}{p^{2}}+\left(-\frac{1}{2}+\frac{1}{3} \cdot \frac{1}{\left(1-\delta_{q}\right)^{2}}\right) \cdot \frac{(\hat{p}-p)^{3}}{q^{2}}\right)\right) \sqrt{\frac{p(1-p)}{\hat{p}(1-\hat{p})}}
\end{aligned}
$$

Finally, $\quad \sup |\tilde{r}(n, k, n-k)| \rightarrow 0, n \rightarrow \infty \quad$ for $k:|k-n p| /(n p q)^{2 / 3} \rightarrow 0$.

The Lemma is proved.

Proof of Lemma 6. Let $\infty<a \leq b<\infty$ $P_{n}(a, b]=\sum_{a<z \leq b} P_{n}\left(n p_{n}+z \sqrt{n p_{n} q_{n}}\right)$, where the sum is over such $\mathrm{z}$ that $n p_{n}+z \sqrt{n p_{n} q_{n}}$ is an integer. From Lemma 5 for each $t_{k}$ such that $k=n p_{n}+t_{k} \sqrt{n p_{n} q_{n}}$ and $\left|t_{k}\right| \leq T<\infty$ :

$$
P_{n}\left(n p_{n}+t_{k} \sqrt{n p_{n} q_{n}}\right)=\frac{\delta_{k}}{\sqrt{2 \pi}} \exp \left(-t_{k}^{2} / 2\right)\left(1+\varepsilon\left(t_{k}, n\right)\right)
$$

where, $\sup _{\left|t_{k}\right| \leq T}\left|\varepsilon\left(t_{k}, n\right)\right| \rightarrow 0, n \rightarrow \infty$ and $\delta_{k}=\frac{1}{\sqrt{n p_{n} q_{n}}}$.

So for the fixed $a$ and $b$ such that $-T \leq a \leq b \leq T, T \leq \infty$ :

$$
\begin{aligned}
& \sum_{a<t_{k} \leq b} P_{n}\left(n p+t_{k} \sqrt{n p q}\right) \\
= & \sum_{a<t_{k} \leq b} \frac{\delta_{k}}{\sqrt{2 \pi}} \exp \left(-t_{k}^{2} / 2\right)+\sum_{a<t_{k} \leq b} \varepsilon\left(t_{k}, n\right) \frac{\delta_{k}}{\sqrt{2 \pi}} \exp \left(-t_{k}^{2} / 2\right) \\
= & \frac{1}{\sqrt{2 \pi}} \int_{a}^{b} \exp \left(-z^{2} / 2\right) d x+R_{n}^{(1)}(a, b)+R_{n}^{(2)}(a, b)
\end{aligned}
$$


Where:

$$
\begin{aligned}
& R_{n}^{(1)}(a, b)=\sum_{a<t_{k} \leq b} \frac{\delta_{k}}{\sqrt{2 \pi}} \exp \left(-t_{k}^{2} / 2\right) \\
& -\frac{1}{\sqrt{2 \pi}} \int_{a}^{b} \exp \left(-z^{2} / 2\right) d x \\
& R_{n}^{(2)}(a, b)=\sum_{a<t_{k} \leq b} \varepsilon\left(t_{k}, n\right) \frac{\delta_{k}}{\sqrt{2 \pi}} \exp \left(-t_{k}^{2} / 2\right) .
\end{aligned}
$$

From the properties of the integral sums $\sup _{-T \leq a \leq b \leq b}\left|R_{n}^{(1)}(a, b)\right| \rightarrow 0, n \rightarrow \infty$.

Given the non-negativity of the integrand:

$$
\frac{1}{\sqrt{2 \pi}} \int_{-T}^{T} \exp \left(-z^{2} / 2\right) d z \leq \frac{1}{2 \pi} \int_{-\infty}^{\infty} \exp \left(-z^{2} / 2\right) d x
$$

That is:

$$
\begin{aligned}
& \sup _{-T \leq a \leq b \leq T}\left|R_{n}^{(2)}(a, b)\right| \leq \sup _{\left|t_{k}\right| \leq T}\left|\varepsilon\left(t_{k}, n\right)\right| \\
& \cdot\left(\frac{1}{\sqrt{2 \pi}} \int_{-T}^{T} \exp \left(-z^{2} / 2\right) d z+\sup _{-T \leq a \leq b \leq T}\left|R_{n}^{(1)}(a, b)\right|\right) \rightarrow 0
\end{aligned}
$$

Denote $\Phi(z)=\frac{1}{\sqrt{2 \pi}} \int_{-\infty}^{z} \exp \left(-t^{2} / 2\right) d t$. Get:

$$
\sup _{-T \leq a \leq b \leq T}\left|P_{n}(a, b]-(\Phi(b)-\Phi(a))\right| \rightarrow 0, n \rightarrow \infty .
$$

Now for $\mathrm{T}=\propto$ : $\forall \varepsilon>0 \quad \exists T=T(\varepsilon)>0$ $\frac{1}{\sqrt{2 \pi}} \int_{-T}^{T} \exp \left(-z^{2} / 2\right) d z>1-\frac{\varepsilon}{4}$.

From (28):

$\exists N: \forall n>N: \sup _{-T \leq a \leq b \leq T}\left|P_{n}(a, b]-(\Phi(b)-\Phi(a))\right|<\frac{\varepsilon}{4}$

So $P_{n}(-T, T]>1-\frac{\varepsilon}{2}, \quad P_{n}(-\infty,-T]+P_{n}[T, \infty)<\frac{\varepsilon}{2}$, where $P_{n}(-\infty,-T]=\lim _{S \rightarrow-\infty} P_{n}(S,-T]$ и $P_{n}(T, \infty)=\lim _{S \rightarrow-\infty} P_{n}[T, S)$.

Finally $\forall T:-\infty \leq-T \leq a \leq b \leq \infty$ :

$$
\begin{aligned}
& \left|P_{n}(a, b]-\frac{1}{\sqrt{2 \pi}} \int_{a}^{b} \exp \left(-z^{2} / 2\right) d z\right| \\
& \leq\left|P_{n}(-T, T]-\frac{1}{\sqrt{2 \pi}} \int_{-T}^{T} \exp \left(-z^{2} / 2\right) d z\right| \\
& +\left|P_{n}(a,-T]-\frac{1}{\sqrt{2 \pi}} \int_{a}^{-T} \exp \left(-z^{2} / 2\right) d z\right| \\
& +\left|P_{n}(T, b]-\frac{1}{\sqrt{2 \pi}} \int_{T}^{b} \exp \left(-z^{2} / 2\right) d z\right| \leq \frac{\varepsilon}{4}+P_{n}(-\infty,-T] \\
& +\frac{1}{\sqrt{2 \pi}} \int_{-\infty}^{-T} \exp \left(-z^{2} / 2\right) d z+P_{n}(T, \infty) \\
& +\frac{1}{\sqrt{2 \pi}} \int_{T}^{\infty} \exp \left(-z^{2} / 2\right) d z \leq \frac{\varepsilon}{4}+\frac{\varepsilon}{2}+\frac{\varepsilon}{8}+\frac{\varepsilon}{8}=\varepsilon .
\end{aligned}
$$

That is $P_{n}(a, b] \rightarrow \Phi(b)-\Phi(b)$ for all $-\infty \leq a<b \leq \infty$. The Lemma is proved.

The Probability of Large Deviations for Bounded Random Variables

Theorem 6. (from Petrov (1987)). Let $x_{1}, \ldots, x_{n}$ be independent random variables, $\mathbb{E} \chi_{k}=0$, $\sigma_{k}^{2}=\mathbb{E} \chi_{k}^{2}<\infty, k=1, \ldots, n, B=\sum_{k=1}^{n} \sigma_{k}^{2}$. Let $H>0$ be such constant that $\left|\mathbb{E} \chi_{k}^{m}\right| \leq \frac{m !}{2} \cdot \sigma_{k}^{2} \cdot H^{m-2}, k=1, \ldots, n$ for all integer $m \geq 2$. Let $S=\sum_{k=1}^{n} \chi_{k}$ then for $0 \leq x \leq B / H$ :

$$
\begin{aligned}
& P(S \geq x) \leq \exp \left(-x^{2} / 4 B\right) \\
& P(S \leq-x) \leq \exp \left(-x^{2} / 4 B\right)
\end{aligned}
$$

and for $x \geq B / H$ :

$$
\begin{aligned}
& P(S \geq x) \leq \exp (-x / 4 H) \\
& P(S \leq-x) \leq \exp (-x / 4 H)
\end{aligned}
$$

We prove the lemma about the probability of large deviations for bounded random variables:

Proof of Lemma 7. Modify:

$$
\begin{aligned}
& \bar{\chi}=\frac{1}{n} \sum_{k=1}^{n} \chi_{k}-m_{k}=\frac{1}{n} \sum_{k=1}^{n}\left(\chi_{k}-\mathbb{E} \chi_{k}\right)+\left(\mathbb{E} \chi_{k}-m_{k}\right) \\
& =\frac{1}{n} \sum_{k=1}^{n}\left(\chi_{k}-\mathbb{E} \chi_{k}\right)+\frac{1}{n} \sum_{k=1}^{n}\left(\mathbb{E} \chi_{k}-m_{k}\right)
\end{aligned}
$$

For the first sum multiplied by $\mathrm{t}$, we use Theorem 6 . To do this, check the conditions of the theorem:

$$
\begin{aligned}
& \mathbb{E}\left(\chi_{k}-\mathbb{E} \chi_{k}\right)=0 \\
& \mathbb{E}\left(\chi_{k}-\mathbb{E} \chi_{k}\right)^{2}=\sigma^{2}<\infty \\
& \left|\mathbb{E}\left(\chi_{k}-\mathbb{E} \chi_{k}\right)^{m}\right| \leq \mathbb{E}\left|\chi_{k}-\mathbb{E} \chi_{k}\right|^{m} \\
& \leq \mathbb{E}\left|\chi_{k}-\mathbb{E} \chi_{k}\right|^{2} \cdot C^{m-2} \\
& \leq \sigma^{2} \cdot C^{m-2} \leq \frac{m !}{2} \sigma^{2} C^{m-2}
\end{aligned}
$$

Thus, the conditions of Theorem 6 fulfilled for $H \geq 2 C$. For $\chi_{1}-\mathbb{E} \chi_{1}, \ldots, \chi_{n}-\mathbb{E} \chi_{n}$ using $\left|\frac{1}{n} \sum_{k=1}^{n}\left(\chi_{k}-\mathbb{E} \chi_{k}\right)-\bar{\chi}\right| \leq m$ we get the result. The Lemma is proved.

\section{Integration Area Replacement}

Proof of Lemma 8. We transform difference of considered in the lemma integrals for $\varepsilon<\frac{1}{c(\mathbb{X})}$ : 


$$
\begin{aligned}
& \int_{B_{\varepsilon}(X)} g(X, \tilde{X}) d V(\tilde{X})-\int_{\tilde{B}_{\varepsilon}(X)} g(X, \tilde{X}) d V(\tilde{X}) \\
& =\int_{B_{\varepsilon}(X) \backslash \tilde{B}_{\varepsilon}(X)} g(X, \tilde{X}) d V(\tilde{X}) \\
& -\int_{\tilde{B}_{\varepsilon}(X) \backslash B_{\varepsilon}(X)} g(X, \tilde{X}) d V(\tilde{X}) .
\end{aligned}
$$

From Lemma 1 follows that each element from $B_{\varepsilon}(X) \Delta \tilde{B}_{\varepsilon}(X)$ is not far from $\tilde{B}_{\varepsilon}(X)$ as $\tilde{\varepsilon}=\frac{1}{24}\left\|I I_{\tilde{X}}(\tilde{\theta}, \tilde{\theta})\right\| \varepsilon^{3} \quad$ for $\quad$ some $\quad \tilde{X} \in \mathbb{X} \quad$ and $\tilde{\theta} \in T_{\tilde{X}}(\mathbb{X}):\|\theta\|=1$. From Lemma 3: $\tilde{\varepsilon} \leq \frac{1}{24} C_{I I} \varepsilon^{3}$.

For $\varepsilon \leq \sqrt{\frac{24 \cdot(\sqrt[q]{2}-1)}{\max \left\{1, C_{I I}\right\}}}:(\varepsilon+\tilde{\varepsilon})^{q} \leq 2 \cdot \varepsilon^{q}$.

For $\varepsilon \leq \frac{1}{2 \sqrt{C_{R i c}}}: 1+C_{R i c}(\varepsilon+\tilde{\varepsilon})^{2} \leq 2$.

Using Lemmas 2 and 4:

$$
\begin{aligned}
& \left|\int_{B_{\varepsilon}(X)} g(X, \tilde{X}) d V(\tilde{X})-\int_{\tilde{B}_{\varepsilon}(X)} g(X, \tilde{X}) d V(\tilde{X})\right| \\
& \leq\left|\int_{\tilde{B}_{\varepsilon+\tilde{\varepsilon}}(X)} g(X, \tilde{X}) d V(\tilde{X})-\int_{\tilde{B}_{\varepsilon-\tilde{\varepsilon}}(X)} g(X, \tilde{X}) d V(\tilde{X})\right| \\
& \leq\left((\varepsilon+\tilde{\varepsilon})^{q}-(\varepsilon-\tilde{\varepsilon})^{q}\right) \cdot V_{q} \\
& \cdot \sup _{X, \tilde{X}}|g(X, \tilde{X})| \cdot\left(1+(\varepsilon+\tilde{\varepsilon})^{2} \cdot C_{R i c}\right) \\
& \leq V_{q} \cdot \sup _{X, \tilde{X}}|g(X, \tilde{X})| \cdot \tilde{2} \varepsilon \cdot\left(1+4 \cdot \varepsilon^{2} \cdot C_{R i c}\right) \\
& \cdot \sum_{m=0}^{q-1} \cdot(\varepsilon+\tilde{\varepsilon})^{m} \cdot(\varepsilon-\tilde{\varepsilon})^{q-m-1} \\
& \leq 2 \cdot V_{q} \cdot \sup _{X, \tilde{X}}|g(X, \tilde{X})| \cdot \tilde{\varepsilon} \cdot q \cdot(\varepsilon+\tilde{\varepsilon})^{q-1}\left(1+4 \cdot \varepsilon^{2} \cdot C_{R i c}\right) \\
& \leq \varepsilon^{q+2} \cdot 2 \cdot V_{q} \cdot\left(1+\frac{1}{24} C_{I I} \varepsilon^{2}\right)^{q} \cdot\left(1+4 \cdot \varepsilon^{2} \cdot C_{R i c}\right) \cdot \sup _{X, \tilde{X}}|g(X, \tilde{X})| \\
& \leq 8 \cdot V_{q} \cdot \sup _{X, \tilde{X}}|g(X, \tilde{X})| \cdot \varepsilon^{q+2}
\end{aligned}
$$

The Lemma is proved.

\section{Finite Nets}

Lemma 10. For each $\delta$ exists finite $\delta$-net on a manifold $\mathbb{X}$ with $\left(\frac{2 a \sqrt{p}}{\delta}\right)^{p}$ or fewer elements, where $a>0$ is an edge of circumscribed $\mathrm{p}$-dimensional hypercube.

Proof. Thus $\mathbb{X} \subset \mathbb{R}^{p}$ is bounded it could be placed into a hypercube $C_{a}$ with edge $a>0$. Let $\delta>0$ be a fixed number. Consider a uniform grid $\mathrm{G}(\delta)$ for the cube with the distances between points along each edge at most $\delta / \sqrt{p}$. The number of points in the grid does not exceed $\left(\frac{a \sqrt{p}}{\delta}\right)^{p}$. Cube $C_{a}$ is divided by net $\mathrm{G}(\delta)$ into small cubes with edges not exceeding $\delta / \sqrt{p}$.
Therefore for every point $Z$ of the cube $G(\delta)$ the distance between the and does not exceed the length of the diagonal of the small cube, which it belongs to:

$$
d(Z, G(\delta)) \leq \sqrt{\sum_{k=1}^{p} \frac{\delta^{2}}{p}}=\delta
$$

where, $d(Z, A)=\inf _{Z^{\prime} \in A}\left\|Z-Z^{\prime}\right\|$ is a distance between point $Z \in \mathbb{R}^{p}$ and set $A \subset \mathbb{R}^{p}$.

Therefore on a manifold $\mathrm{G}(\delta)$ for every point $X \in \mathbb{X}$ contains a point distant from it by no more than $\delta$. But $G(\delta) \not \subset \mathbb{X}$.

Denote the ball with center $\mathrm{X}$ and radius $\delta$ as $B_{\delta}(X)$. Denote $\tilde{G}(\delta)$ : For each point $X \in G(\delta / 2)$, if $\tilde{B}_{\delta / 2}(X)=B_{\delta}(X) \cap \mathbb{X} \neq \varnothing$, we get $\tilde{X}$ from $\tilde{B}_{\delta}$ and add it to $\tilde{G}(\delta)$. The set $\tilde{G}(\delta)$ is a $\delta$-net for $\mathbb{X}$ and contains not more than $\left(\frac{2 a \sqrt{p}}{\delta}\right)^{p}$ points. The Lemma is proved.

We prove the lemma about the joint occurrence of events system:

Lemma 11. Let each of the events $A_{1}$, dots, $A_{M}$ occurs with a probability of not less than $\mathrm{p}$. Then they all come together with a probability of at least $P\left(\cap_{m=1}^{M} A_{m}\right) \geq 1-M \cdot(1-p)$.

Proof. Let $\bar{A}$ be the complement of a Borel set A to the set of elementary events. Transform:

$$
\begin{aligned}
& \cap_{m=1}^{M} A_{m} \subset \overline{\cap_{m=1}^{M} \bar{A}_{m}} ; P\left(\cap_{m=1}^{M} A_{m}\right) \geq P\left(\overline{\cap_{m=1}^{M} \bar{A}_{m}}\right) \\
& =1-P\left(\cap_{m=1}^{M} \bar{A}_{m}\right) \geq 1-\sum_{m=1}^{M} P\left(\bar{A}_{m}\right) \geq 1-M \cdot(1-p)
\end{aligned}
$$

The Lemma is proved.

\section{References}

Belkin, M. and P. Niyogi, 2003. Laplacian eigenmaps for dimensionality reduction and data representation. J. Neural Computation, 15: 1373-1396. DOI: $10.1162 / 089976603321780317$

Bengio, Y., A. Courville and P. Vincent, 2013. Representation learning: A review and new perspectives. IEEE Transactions Pattern Analysis Machine Intelligence, 35: 1798-1828. DOI: 10.1109/TPAMI.2013.50

Bengio, Y., J.F. Paiement and P. Vincent, 2003. Out-ofsample extensions for LLE, Isomap, MDS, eigenmaps and spectral clustering. Proceedings of the Advances in Neural Information Processing Systems, (ANI' 03), pp: 177-184. 
Bernstein, A. and A. Kuleshov, 2014. Low-Dimensional Data Representation in Data Analysis. In: Artificial Neural Networks in Pattern Recognition, Mana, N., F. Schwenker and E. Trentin, (Eds.), Springer Berlin Heidelberg, Berlin, ISBN-10: 3642332110, pp: 47-58.

Campadelli, P., E. Casiraghi, C. Ceruti and A. Rozza, 2015. Intrinsic dimension estimation: Relevant techniques and a benchmark framework. Mathematical Problems Eng., 2015: 1-21.

DOI: $10.1155 / 2015 / 759567$

Coifman, R.R. and S. Lafon, 2006. Diffusion maps. Applied Comput. Harmonic Analysis, 21: 5-30. DOI: $10.1016 /$ j.acha.2006.04.006

Cox, M.A.A. and T.F. Cox, 2008. Multidimensional Scaling. In: Handbook of Data Visualization, Berlin, Heidelberg: Springer Berlin Heidelberg, pp: 315-347.

Donoho, D.L., 2000. High-dimensional data analysis: The curses and blessings of dimensionality. Proceedings of the AMS Conference on Math Challenges of 21 st Century, (CMC' 00), pp: 1-31.

Donoho, D.L. and C. Grimes, 2003. Hessian eigenmaps: Locally linear embedding techniques for high-dimensional data. Proc. Nati. Acad. Sci., 100: 5591-5596. PMID: 16576753

Einbeck, J. and Z. Kalantana, 2013. Intrinsic dimensionality estimation for high-dimensional data sets: New approaches for the computation of correlation dimension. J. Emerg. Technol. Web Intelli., 5: 91-97. DOI: 10.4304/jetwi.5.2.91-97

Fan, M., H. Qiao and B. Zhang, 2009. Intrinsic dimension estimation of manifolds by incising balls. Patt. Recognit., 42: 780-787.

DOI: 10.1016/j.patcog.2008.09.016

Farahmand, A.M., C. Szepesvári and J.Y. Audibert, 2007. Manifold-adaptive dimension estimation. Proceedings of the 24th International Conference on Machine Learning (ICML '07), New York, pp: 265-272.

Goldberg, Y., A. Zakai, D. Kushnir and Y. Ritov, 2008. Manifold learning: The price of normalization. J. Mach. Learn. Re., 9: 1909-1939.

Jolliffe, I.T., 2002. Principal Component Analysis. 1st Edn., Springer Science and Business Media, New York, ISBN-10: 0387954422, pp: 487.

Kuleshov, A. and A. Bernstein, 2016. Extended regression on manifolds estimation. Proceedings of the 5th International Symposium on Conformal and Probabilistic Prediction with Applications, (ISC' 16), New York, pp: 208-228.

DOI: $10.1007 / 978-3-319-33395-3 \_15$

Kuleshov, A. and A. Bernstein, 2014. Manifold Learning in Data Mining Tasks. In: Machine Learning and Data Mining in Pattern Recognition, Perner, P. (Ed.), Springer, Cham, ISBN-10: 331908979X, pp: 119-133.

Lee, J.A. and M. Verleysen, 2007. Nonlinear Dimensionality Reduction. 1st Edn., Springer Science and Business Media, ISBN-10: 038739351X, pp: 309.
Levina, E. and P.J. Bickel, 2005. Maximum likelihood estimation of intrinsic dimension. Proceedings of the Advances in Neural Information Processing Systems, (ANI' 05), MIT Press, pp: 777-784.

Ma, Y. and Y. Fu, 2011. Manifold Learning Theory and Applications. 1st Edn., CRC Press, Boca Raton, ISBN-10: 1466558873, pp: 314.

Niyogi, P., S. Smale and S. Weinberger, 2008. Finding the homology of submanifolds with high confidence from random samples. Discrete Computational Geometry, 39: 419-441.

Pennec, X., 1999. Probabilities and Statistics on Riemannian Manifolds: Basic Tools For Geometric Measurements. Proceedings of the Workshop on Nonlinear Signal and Image Processing, Jun. 20-23, Antalya, Turkey, pp: 194-198.

Petersen, P., 2006. Riemannian Geometry. Springer New York.

Petrov, V.V., 1987. Limit theorems for sums of independent random variables. Moscow, Nauka.

Roweis, S.T. and L.K. Saul, 2000. Nonlinear dimensionality reduction by locally linear embedding. Science, 290: 2323-2326.

Rozza, A., G. Lombardi, M. Rosa, E. Casiraghi and P. Campadelli, 2011. IDEA: Intrinsic dimension estimation algorithm. Proceedings of the 16th International Conference, Sep. 14-16, Ravenna, Italy, pp: 433-442. DOI: 10.1007/978-3-642-24085-0_45

Saul, L.K., S.T. Roweis and Y. Singer, 2003. Think globally, fit locally: Unsupervised learning of low dimensional manifolds. J. Machine Learning Research, 4: 119-155.

Seung, H.S. and D.D. Lee, 2000. Cognition. The manifold ways of perception. Science, 290: 2268-2269. PMID: 11188725

Shiryayev, A.N., 1984. Probability. 1st Edn., SpringerVerlag, New York.

Singer, A. and H.T. Wu, 2012. Vector diffusion maps and the connection Laplacian. Commun. Pure Applied Math., 65: 1067-1144. DOI: $10.1002 /$ cpa.21395FVAC

Smith, A., X. Huo and H. Zha, 2008. Convergence and Rate of Convergence of a Manifold-Based Dimension Reduction Algorithm. Proceedings of the Proceedings of the Twenty-Second Annual Conference on Neural Information Processing Systems, Dec. 8-11, Vancouver, British Columbia, pp: 1529-1536.

Smith, A., H. Zha and X. Wu, 2009. Convergence and Rate of Convergence of a Manifold-Based Dimension Reduction Algorithm. Advances Neural Information Proc. Sys., 21: 1529-1536.

Tenenbaum, J.B., V. de Silva and J. Langford, 2000. A global geometric framework for nonlinear dimensionality reduction. Science, 290: 2319-2323. DOI: $10.1126 /$ science.290.5500.2319 
Verleysen, M., 2003. Learning high-dimensional data. Limitations Future Trends Neural Computation, 186: 141-162.

Weinberger, K.Q. and L.K. Saul, 2006. Unsupervised learning of image manifolds by semidefinite programming. Int. J. Comput. Vision, 70: 77-90.
Zhang, Z. and H. Zha, 2004. Principal manifolds and nonlinear dimensionality reduction via tangent space alignment. SIAM J. Sci. Comput., 26: 313-338. 